\title{
A Study on Volatility Spurious Almost Integration Effect: A Threshold Realized GARCH Approach
}

\author{
Dinghai $\mathrm{Xu}^{*}$
}

\begin{abstract}
This paper investigates the "spurious almost integration" effect of volatility under a threshold GARCH structure with realized volatility measures. To closely examine the effect, the realized persistence of volatility is proposed to be used as a threshold trigger for volatility regimes. Under the threshold framework, general closed-form solutions of moment conditions are derived, which provide a convenient way to theoretically examine the "spurious almost integration" effect and its associated impacts. We find that introducing the volatility persistence-driven threshold can capture regime-specific characteristics well. It performs better than the traditional GARCH-type models in terms of both in-sample fitting and out-of-sample forecasting. Based on our Monte Carlo and empirical results, in general we find that overlooking the relatively low persistence regime(s) could lead to some misleading conclusions.
\end{abstract}

Keywords: Volatility Persistence; Spurious Almost Integration; Threshold Realized GARCH Model; Realized Kernel; Value at Risk.

JEL: C01, C58

*Department of Economics, University of Waterloo, Waterloo, Ontario, N2L 3G1, Canada. Email: dhxu@uwaterloo.ca; Tel: 001-519-888-4567 ext. 32047. 


\section{Introduction}

Volatility of asset returns plays an important role in many areas of financial research. Volatility modeling literature has grown rapidly in the past three decades since the birth of the (Generalized) Autoregressive Conditional Heteroscedasticity (ARCH/GARCH) model by Engle (1982) and Bollerslev (1986). In essence, under the traditional ARCH/GARCH or its extended specifications, the time-varying latent volatility is typically modeled as a certain function of historical returns (for instance, squared returns) and past volatilities. GARCH models have been widely used and applied in both academia and industry. As is well known, GARCH models can well capture the persistence behavior of the conditional variances. For instance, in the standard GARCH setting, the sum of the estimated autoregressive ARCH and GARCH coefficients is typically found to be close to one for most of the financial asset return data. However, this "stylized" phenomenon should be carefully investigated especially under structural changes in the data. This concern was first raised by Diebold (1986). Lamoureux and Lastrapes (1990) illustrate an overestimation effect of volatility persistence by allowing for different regime values for the constant term in the GARCH conditional volatility equation. A more detailed investigation on this issue is carried out in Hillebrand (2005), which finds that even a simple parameter-shift in the conditional volatility generating process could severely bias the overall persistence estimation toward one. This is independent of estimation approach and the characteristics/properties of the parameter shifts. Hillebrand (2005) provides both theoretical and Monte Carlo results to illustrate this so-called "spurious almost-integration" effect. These concerns raise an interesting and serious question that the commonly-believed highly persistent volatility behavior implied from the conventional volatility models (such as GARCH) may be just due to a mis-specification. As Hillebrand (2005) points out, a regime switch structure could be a natural consideration to investigate this problem.

In recent years, with more and more intra-daily high frequency trading data available for research, there has been considerable interest in incorporating the realized measures of volatility into the traditional volatility model framework. Andersen et al. (2003) and Hansen et al. (2012) point out that one new key feature of this class of models is accommodating and updating new information at a faster speed than the conventional volatility models. Therefore, the models with realized volatility measures provide a better structure in modeling the dynamics of the latent return volatility. Engle and Gallo (2006) first introduce the realized measures in the GARCH process, known as the Multiplicative Error Model (MEM). Hansen et al. (2012) propose a socalled realized GARCH (RGARCH), in which a measurement equation is built into the process to link the latent volatility and the corresponding realized measure. Hansen and Huang (2016) extend the RGARCH to an Exponential GARCH structure to better capture the dependence between the returns and volatility. Takahashi et al. (2009) introduce the comparable version of the RGARCH model in Hansen et al. (2012) under the context of stochastic volatility (SV) framework, referred as realized SV model. In the realized SV, the conditional volatility is modeled to follow a stochastic process with a bridge equation to its realized measures constructed from high frequency data. Chaussé and Xu (2016) generalize the realized SV model accommodating a more flexible correlation structure. Some theoretical model properties are investigated and examined in the context of Generalized Method of Moments (GMM). This literature has grown rapidly in the past decade, see Bollerslev and Zhou (2006), Corradi and Distaso (2006), Dobrev and Szerszen (2010), Shephard and Sheppard (2010), Noureldin et al. (2012), Koopman and Scharth (2013) and references therein.

Volatility models with realized measures, such as RGARCH, have many attractive character- 
istics and provide better performance in both sample fitting and forecasting than the traditional volatility models. However, we find that the "spurious almost integration" effect is still embedded in the RGARCH structure. In other words, even a single changing point in the parameters in data generating process could cause an overestimation of the true persistence of the volatility. Hansen et al. (2012) investigate 28 stocks and 1 stock index under the proposed RGARCH model. There is an interesting "common" finding on the results across all 29 assets. The volatility persistence is typically found to be high, for example, in the $\log$-linear $\operatorname{RGARCH}(1,2)$ results, the average persistence over these 29 assets is 0.99 . Using a "half-life" measure of the volatility shocks, this persistence value means that on average it takes about 70 days that a shock to current volatility level diminishes to half of its initial size 1 Is it a "stylized fact" or is it simply due to the presence of certain structure break in the sample data? We believe that it should deserve a further investigation to avoid some misleading conclusions. Motivated by this interesting phenomenon, in this paper, we develop a threshold triggered regime-switch RGARCH model. Regime switch, especially the threshold triggered regime switch, has been introduced into volatility modeling in the literature. See Glosten et al. (1993), Hamilton and Susmel (1994), Zakoian (1994), Li and Lam (1995), Brooks (2001), Chen and So (2006), Xu (2013) and references therein. In the literature, the popularly used candidate threshold variable is the asset return. In this paper, we propose to use volatility persistence as a candidate threshold variable to determine the volatility regimes. To the best of our knowledge, this paper is the first one to adopt persistence as an indicator for volatility dynamics. This is motivated by the findings from Ning et al. (2015), which detect strong evidence of asymmetric patterns in volatility clustering. In particular, clusters of high volatility occur more frequently than clusters of low volatility. However, unlike the asset prices (returns), the volatility is latent, so is its persistence. In this paper, the volatility persistence estimation is conducted over a rolling window of the realized kernel estimates of the volatility. As is well known, measurement errors could potential deteriorate the quality of the estimates of the persistence. In this paper, following Hansen and Lunde (2014), we adopt a non-parametric instrumental variable (IV)-based method to construct time-varying persistence estimates, which are treated as important indicators for the volatility regimes.

In general, both our Monte Carlo results and empirical evidence support introducing the threshold effect into modelling the volatility dynamics. The proposal of using the volatility persistence to trigger the regime-switch provides a more intuitive economic interpretation of the structure. In the case of a two-regime model, we empirically find that the high persistence regime is normally associated with high volatility level, while low persistence regime is normally associated with low volatility level. In addition, we find improvements of the proposed model in both in-sample fitting and out-of-sample forecasting comparing to the conventional benchmark (without threshold effect). More accurate risk measures are also implied under the threshold structure.

The rest of the paper is organized as follows. Section 2 introduces the threshold structure under a realized GARCH framework (TRGARCH). General closed form moment conditions are derived for investigation on the volatility "spurious almost integration" effect and its associated impacts. In section 3, several groups of Monte Carlo simulations are conducted. Section 4 provides empirical applications consisting of two groups of sample data. Section 5 concludes. All the proofs are provided in Appendix A and some tables and figures are collected in Appendix B.

\footnotetext{
${ }^{1}$ We will discuss this "half-life" measure in the empirical applications (Section 4) in more details. Here we also provide a "quarter life" measure for readers' reference. At the persistence level of 0.99 , it takes about 138.9 days for a shock to diminish to a quarter of its initial impact to volatility.
} 


\section{General Structure of a Threshold Realized GARCH Model}

Following Hansen et al. (2012), a standard $\operatorname{RGARCH}(p, q)$ has the following specification,

$$
\begin{aligned}
x_{t} & =\exp \left(h_{t} / 2\right) \epsilon_{t} \\
y_{t} & =\beta_{1}+\beta_{2} h_{t}+\tau\left(\epsilon_{t}\right)+u_{t} \\
h_{t} & =\lambda+\sum_{i=1}^{p} \alpha_{i} h_{t-i}+\sum_{j=1}^{q} \gamma_{j} y_{t-j}
\end{aligned}
$$

There are three equations in the system. (11) is referred as return process, where $x_{t}$ is the continuously compounded return time series, defined by the logarithm of the ratio of two consecutive prices. $h_{t}$ is the latent logarithmic volatility at time $t . y_{t}$ is the corresponding realized measure for $h_{t}$. It can be constructed from the high frequency trading price data. $\tau\left(\epsilon_{t}\right)$ is referred as the so-called leverage function, which captures the correlation between the return and volatility. Following the specification in Hansen et al. (2012), we adopt the convenient functional form of a Hermite polynomials with the first two orders, that is, $\tau\left(\epsilon_{t}\right)=\tau_{1} \epsilon_{t}+\tau_{2}\left(\epsilon_{t}^{2}-1\right)$. (2) serves the role as a measurement equation linking the latent volatility and the realized measures.(3) is the standard $\operatorname{GARCH}(p, q)$-like equation modeling the dynamics of the volatility. $\epsilon_{t}$ is typically assumed with $\operatorname{NID}(0,1)$ and $u_{t} \sim \operatorname{NID}\left(0, \sigma^{2}\right)$.

In this paper, we extend the above structure into a threshold heteroscedastic model triggered by a regime-indicator variable $\rho$. Suppose that there are $K$ regimes in the volatility space. Let $s(\rho)=s_{k}$ with $k=1,2, \ldots, K$. In each regime, the measurement between the volatility and realized measures and the volatility dynamics would exhibit different behavior. In other words, (2) becomes,

$$
\begin{array}{rlr}
y_{t} & =\beta_{s_{1}, 1}+\beta_{s_{1}, 2} h_{t}+\tau_{s_{1}, 1} \epsilon_{t}+\tau_{s_{1}, 2}\left(\epsilon_{t}^{2}-1\right)+u_{s_{1}, t} & s(\rho)=s_{1} \\
y_{t}=\beta_{s_{2}, 1}+\beta_{s_{2}, 2} h_{t}+\tau_{s_{2}, 1} \epsilon_{t}+\tau_{s_{2}, 2}\left(\epsilon_{t}^{2}-1\right)+u_{s_{2}, t} & s(\rho)=s_{2} \\
& \cdots & \\
y_{t}=\beta_{s_{K}, 1}+\beta_{s_{K}, 2} h_{t}+\tau_{s_{K}, 1} \epsilon_{t}+\tau_{s_{K}, 2}\left(\epsilon_{t}^{2}-1\right)+u_{s_{K}, t} & s(\rho)=s_{K}
\end{array}
$$

Correspondingly, (3) becomes,

$$
\begin{array}{rlrl}
h_{t} & =\lambda_{s_{1}}+\sum_{i=1}^{p} \alpha_{s_{1}, i} h_{t-i}+\sum_{j=1}^{q} \gamma_{s_{1}, j} y_{t-j} & s(\rho)=s_{1} \\
h_{t} & =\lambda_{s_{2}}+\sum_{i=1}^{p} \alpha_{s_{2}, i} h_{t-i}+\sum_{j=1}^{q} \gamma_{s_{2}, j} y_{t-j} & s(\rho)=s_{2} \\
& \cdots & \\
h_{t} & =\lambda_{s_{K}}+\sum_{i=1}^{p} \alpha_{s_{K}, i} h_{t-i}+\sum_{j=1}^{q} \gamma_{s_{K}, j} y_{t-j} & s(\rho)=s_{K}
\end{array}
$$

Therefore, (10), (4) and (5) define a general structure of a K-regime realized GARCH model. The coefficients in (4) and (5) are all state-dependent, which could capture and explain regimespecific characteristics. In particular, the unknowns for the K-regime realized GARCH $(p, q)$ model are collected in the $\theta$-vector, where $\theta=\left(\beta_{s_{k}, 1}, \beta_{s_{k}, 2}, \tau_{s_{k}, 1}, \tau_{s_{k}, 2}, \lambda_{s_{k}}, \alpha_{s_{k}, i}, \gamma_{s_{k}, j}, \sigma_{s_{k}}^{2}\right)$, with 
$k=1,2, \ldots, K, i=1,2, \ldots, p$ and $j=1,2, \ldots, q$. There are, in total, $(6+p+q) K$ parameters.

Proposition 1. Given the K-regime realized GARCH $(p, q)$ model specified in (1), (4) and (5), when $p=1$ and $q=1$, the overall persistence of the volatility (denoted as $\omega$ ) and the leverage effect (denoted as $\varphi$ ) are implied as follows,

$$
\begin{aligned}
\omega & =\sum_{k=1}^{K} p\left(s(\rho)=s_{k}\right)\left(\alpha_{s_{k}}+\gamma_{s_{k}} \beta_{s_{k}, 2}\right) \\
\varphi & =\sum_{k=1}^{K} p\left(s(\rho)=s_{k}\right) \frac{\tau_{s_{k}, 1}}{\sqrt{\tau_{s_{k}, 1}^{2}+2 \tau_{s_{k}, 2}^{2}+\sigma_{s_{k}}^{2}}}
\end{aligned}
$$

Proof. see Appendix A.2

Proposition 2. Given the K-regime realized $G A R C H(p, q)$ model specified in (1), (4) and (5), when $p=1$ and $q=1$, the Moment Generating Function (MGF) for $h_{t}$ and $y_{t}$ are provided as follows,

$$
\begin{aligned}
& M_{h}(\eta)=\sum_{k=1}^{K} p\left(s(\rho)=s_{k}\right) \exp \left(\frac{\eta \omega_{s_{k}, 1}}{1-\omega_{s_{k}, 2}}\right) \prod_{j=0}^{\infty} M_{v}\left(\eta \omega_{s_{k}, 2}^{j}\right) \\
& M_{y}(\eta)=\sum_{k=1}^{K} p\left(s(\rho)=s_{k}\right) M_{\zeta}(\eta) M_{\epsilon^{2}}(\eta) M_{h_{k}}\left(\eta \beta_{s_{k}, 2}\right)
\end{aligned}
$$

Proof. see Appendix A. $M_{\psi}($.$) represents the MGF of \psi$. They are all defined in the proof.

Proposition 3. Given the K-regime realized GARCH (p,q) model specified in (1), (4) and (5), when $p=1$ and $q=1$, the general moments for $x_{t}$ are specified as follows,

$$
E\left(x_{t}^{m}\right)=\frac{\partial^{m} M_{\epsilon}(\eta)}{\partial \eta^{m}} \|_{\eta=0}\left(\sum_{k=1}^{K} p\left(s(\rho)=s_{k}\right) M_{h_{k}}\left(\frac{m}{2}\right)\right)
$$

More generally, the $(m, n)$ th order cross-moment of $x_{t}$ and $x_{t+g}$ has the following closed-form,

$$
\begin{aligned}
E\left(x_{t}^{m} x_{t+g}^{n}\right) & =\left[\sum_{k=1}^{K} p\left(s(\rho)=s_{k}\right) M_{h_{k}}\left(\frac{m+n \omega_{s_{k}, 2}^{g}}{2}\right) \exp \left(\frac{n}{2} \omega_{s_{k}, 1} \sum_{j=1}^{g} \omega_{s_{k}, 2}^{j-1}\right)\right. \\
& \left.\times \prod_{j=1}^{g} M_{v}\left(\frac{n}{2} \omega_{s_{k}, 2}^{(g-j)}\right)\right] \frac{\partial^{m} M_{\epsilon}(\eta)}{\partial \eta^{m}}\left\|_{\eta=0} \frac{\partial^{n} M_{\epsilon}(\eta)}{\partial \eta^{n}}\right\|_{\eta=0}
\end{aligned}
$$

Proof. see Appendix A. $M_{\psi}($.$) represents the MGF of \psi \cdot \frac{\partial^{i} f(\xi)}{\partial \xi^{m}} \|_{\xi=0}$ stands for the $i$ th derivative of $f($.$) w.r.t \xi$ evaluated at $\xi=0$.

(8), (9), (10) and (11) in Proposition 2 and 3 can theoretically supply any order of moments of the return, latent volatility and the realized measure implied from the model. More importantly, these closed form solutions provide a convenient way to further investigate the theoretical properties of the model. For instance, the impact of the so-called "spurious almost integration"

\footnotetext{
${ }^{2}$ In Proposition 1, we only present the persistence and leverage effect in the case of $p=1$ and $q=1$. For any other order, it is a straightforward extension based on the proof in Appendix.
} 
effect (overestimation of the volatility persistence) on the variance and kurtosis of return can be seen via the corresponding moments's plots against relevant parameters. For illustration, we take a set of empirical estimates of the RGARCH(1,1) on S\&P 500 index in Hansen et al. (2012). $\hat{\theta}=\left(\hat{\lambda}, \hat{\alpha}, \hat{\gamma}, \hat{\beta}_{1}, \hat{\beta}_{2}, \hat{\tau_{1}}, \hat{\tau_{2}}, \hat{\sigma_{u}}\right)=(0.06,0.55,0.41,-0.18,1.04,-0.07,0.07,0.38)$. In Figure 1 and 2. we investigate the impact of each parameter on the variance and kurtosis of returns by fixing other parameter values. In particular, we pay attention to the two key parameters in the persistence, $\alpha$ and $\gamma 3$ As one can see from Figure 1 and 2, both $\alpha$ and $\gamma$ impact the return variance and kurtosis in the same direction. More specifically, overestimating the persistence can lead to underestimation of return variance and overestimation of return kurtosis. This is also supported by Figure 3, which plots the 3-dimension relationship of $\alpha$ and $\gamma$ on return variance (upper panel) and kurtosis (lower panel). Taking the advantage of close-form solution in (11), we can further examine how the persistence (via $\alpha$ and $\gamma$ ) impacts on the autocorrelation function (ACF) of the squared returns. Figure 4 presents the ACF plots (over 20 lags) under the persistence changes due to $\alpha$-switch (upper panel) and $\gamma$-switch (lower panel). One can see that overestimating the persistence could lead to a positively biased ACF in both $\alpha$-switch case and $\gamma$-switch case. As the volatility persistence approaches closer to 1 ("spurious almost integration"), the bias increases at a faster rate. For instance, in the $\alpha$-switch case, when $\alpha$ changes from 0.1 to 0.3 (equivalent to persistence level from 0.53 to 0.73 ), the ACF is slightly shifted, while when $\alpha$ switches from 0.5 to 0.55 (equivalent to persistence level from 0.93 to 0.98 ), the ACF is shifted significantly. In other words, if the persistence is overestimated (especially towards to one), the ACF of squared returns is positively biased.

The modified Quasi Maximum Likelihood estimator (mQMLE) based on Hansen et al. (2012) are applied in this paper for estimation. In particular, the full likelihood function is defined over a sequential products of the conditional densities. These conditional densities are state dependent determined by the indicator function of the threshold variable. Therefore, under the Gaussian assumption, the objective log-likelihood function is given by,

$$
\ell(x, y, \theta)=-\frac{1}{2} \sum_{t=1}^{T}\left(\ell_{t} \mid I_{t-1}\right)
$$

$\ell_{t}$ is the log-likelihood at time $t$ given the information up to $t-1$. In particular, it can be constructed as follows,

(i) when $s\left(\rho_{t-1}\right)=s_{k}$, it indicates that the volatility dynamics follows the $k$ th regime process;

(ii) given $\left\{y_{t}\right\},\left\{\lambda_{s_{k}}\right\},\left\{\alpha_{s_{k}, i}\right\}_{i=1}^{p},\left\{\gamma_{s_{k}, j}\right\}_{j=1}^{q}$ and initial values of $\{h\}_{t-p}^{t-1}, h_{t}$ can be computed by $\lambda_{s_{k}}+\sum_{i=1}^{p} \alpha_{s_{k}, i} h_{t-i}+\sum_{j=1}^{q} \gamma_{s_{k}, j} y_{t-j}$

(iii) given $\left\{x_{t}, h_{t}\right\}, \epsilon_{t}=x_{t} * \exp \left(-h_{t} / 2\right)$;

(iv) given $\left\{\beta_{s_{k}, 1}, \beta_{s_{k}, 2}, \tau_{s_{k}, 1}, \tau_{s_{k}, 2}\right\}$ and $\left\{\epsilon_{t}\right\}, u_{s_{k}, t}=y_{t}-\beta_{s_{k}, 1}-\beta_{s_{k}, 2} h_{t}-\tau_{s_{k}, 1} \epsilon_{t}-\tau_{s_{k}, 2}\left(\epsilon_{t}^{2}-1\right)$;

(v) $\ell_{t}=\log \left(h_{t}\right)+\log \left(\sigma_{s_{k}}^{2}\right)+x_{t}^{2} / h_{t}+u_{s_{k}, t}^{2} / \sigma_{s_{k}}^{2}$.

Following Straumann and Mikosch (2012) and Hansen et al. (2012), we construct the standard error for the mQMLE in a typical sandwich form that $\sqrt{T}(\hat{\theta}-\theta) \rightarrow N\left(0, \Omega^{-1} \Lambda \Omega^{-1}\right)$, where

\footnotetext{
${ }^{3}$ According to (6), there are three relevant parameters characterizing volatility persistence, $\alpha, \gamma$ and $\beta_{2}$. However, $\beta_{2}$ is found to be stable around 1 empirically, which is also consistent with the theoretical explanation that the realized measures are expected to be proportional to the latent volatility. Hansen and Huang (2016) also discuss imposing this restriction $\left(\beta_{2}=1\right)$ in the framework. In this paper, we examine the persistence impacts through the changes of $\alpha$ and $\gamma$.
} 
$\Omega=E\left(\frac{\partial^{2} \ell}{\partial \theta \partial \theta^{\prime}}\right)$ and $\Lambda=E\left(\left(\frac{\partial \ell}{\partial \theta}\right)\left(\frac{\partial \ell}{\partial \theta}\right)^{\prime}\right)$, with $\frac{1}{\sqrt{T}} \sum_{t=1}^{T} \frac{\partial \ell_{t}}{\partial \theta} \stackrel{d}{\rightarrow} N(0, \Lambda)$ and $-\frac{1}{T} \sum_{t=1}^{T} \frac{\partial^{2} \ell_{t}}{\partial \theta \partial \theta^{\prime}} \stackrel{p}{\rightarrow} \Omega$.

In this paper, we propose to use the realized persistence of volatility as the threshold to trigger the regime switch. It could explain the findings in Ning et al. (2015), which indicates that high persistence is more often observed in the high volatile period, and vice versa. It also contributes to the investigation on the so called "spurious almost integration" effect. In Hansen et al. (2012), high persistence is commonly detected in all their sample data examined. Under our proposed framework, it could explain whether this phenomenon is consistent with the stylized fact in the literature or it is simply due to parameter(s) shift. However, the volatility persistence is latent. A robust way is needed to construct the corresponding approximation. Recently, with more and more trading data available at intra-daily level, there is a growing literature on constructing realized proxies for volatility using these high frequency data, see for example Barndorff-Nielsen and Shephard (2002), Zhang et al. (2005), Barndorff-Nielsen et al. (2008), Barndorff-Nielsen et al. (2011) and references therein. This provides us a solid foundation for the volatility persistence estimation. However, as Hansen and Lunde (2014) point out, the measurement errors can influence the quality of the volatility persistence estimate constructed from the high frequency data (such as realized volatility or realized kernel). Therefore, Hansen and Lunde (2014) develop an IV-based method to estimate the volatility persistence. They demonstrate that the proposed IV estimator has a better finite sample properties.

In this paper, we construct our realized volatility persistence following Hansen and Lunde (2014). In particular, we utilize the multiple instruments method to estimate the persistence parameter with a rolling window on a realized kernel sequence. Suppose $y_{t}$ is the logarithm of the realized kernel measure. Assume that each rolling window has a fixed window size with $n$. Define the multiple instruments as $Z_{t}=\left(y_{t-n_{1}}-\bar{y}_{n_{1}}, \ldots, y_{t-n_{2}}-\bar{y}_{n_{2}}\right)^{\prime}$, where $0 \leq n_{1} \leq n_{2} \leq n$. Then the realized volatility persistence is estimated as follows,

$$
\widehat{\rho_{t}}=\left(\sum_{t=1}^{n-1} Z_{t} y_{t}\right)^{-1}\left(\sum_{t=1}^{n-1} Z_{t} y_{t+1}\right)
$$

The above formula, (13), is just the multiple IV estimator version presented in Hansen and Lunde (2014). For each rolling window, we can achieve a volatility persistence estimate. Therefore, a sequence of $\widehat{\rho_{t}}$ 's can be constructed as thresholds. In particular, this sequence of estimates will be the input of our indicator function to determine the regime with $s(\widehat{\rho})=s_{k}, k=1,2, \ldots K$. Theoretically, we need to use $K-1$ threshold levels, $\left(\rho_{1}^{*}, \rho_{2}^{*}, \ldots, \rho_{K-1}^{*}\right)$, to define $K$ regimes. In other words, $s\left(\rho_{i}^{*} \leq \widehat{\rho}<\rho_{i+1}^{*}\right)=s_{i+1}$, with $i=1, \ldots, K-2$. If $\rho<\rho_{1}^{*}$ or $\rho \geq \rho_{K-1}^{*}$, it will be in regime 1 or $K$ respectively.

\section{Monte Carlo Experiments}

In the beginning of this section, we investigate the so-called "spurious almost integration" effect in the RGARCH structure via Monte Carlo experiments. In the first group of Monte Carlo experiments, the true data generating process (DGP) is specified as follows,

$$
\begin{aligned}
& x_{t}=\exp \left(h_{t} / 2\right) \epsilon_{t} \\
& y_{t}=-0.18+1.04 h_{t}-0.07 \epsilon_{t}+0.07\left(\epsilon_{t}^{2}-1\right)+u_{t} \\
& h_{t}=0.06+\alpha h_{t-1}+\gamma y_{t-1} \quad u_{t} \sim N\left(0,0.38^{2}\right)
\end{aligned}
$$


All the parameter values are taken from the empirical estimates of the RGARCH $(1,1)$ to S\&P 500 returns in Hansen et al. (2012). We allow $\alpha$ or $\gamma$ to be shifted across time as $\alpha$ or $\gamma$ is one component in determining the persistence level. In Hansen et al. (2012), $\alpha=0.55$ and $\gamma=0.41$, correspondingly, the persistence is found to be 0.976, which indicates high persistence behavior of the S\&P volatility empirically. In this experiment, we allow the parameter-shift right on the middle time point. In particular, in the first half of the data, $\alpha=0.10$ (or $\gamma=0.10$ ) and in the second half of the data, $\alpha=0.55$ (or $\gamma=0.41$ ) 4 In the setting, in the first regime, the persistence is low, which is equal to 0.526 (or 0.654). While in the second regime, the true persistence is the same as in Hansen et al. (2012), which is 0.976. Since the switch occurs in the middle of the sample, the true overall persistence is simply the average of the two regimes' persistence, which is 0.751 (or 0.815). The data are then simulated based on (14). The estimation is conducted neglecting the parameter jumps. In other words, we just fit a one-regime RGARCH model to the simulated data. The procedure is replicated 1000 times. The results are presented as follows in Table 1 .

Table 1: Spurious almost integration effects caused by a single parameter shift

\begin{tabular}{c|ccccccccc}
\hline & $\lambda$ & $\alpha$ & $\gamma$ & $\beta_{1}$ & $\beta_{2}$ & $\tau_{1}$ & $\tau_{2}$ & $\sigma$ & $\pi$ \\
\hline$\alpha=0.10$ to 0.55 & 0.0621 & 0.5235 & 0.4125 & -0.1805 & 1.0411 & -0.0695 & 0.0736 & 0.3900 & 0.9501 \\
& $(0.0273)$ & $(0.0471)$ & $(0.0474)$ & $(0.0531)$ & $(0.0837)$ & $(0.0124)$ & $(0.0094)$ & $(0.0093)$ & $(0.0234)$ \\
$\gamma=0.10$ to 0.41 & 0.0503 & 0.6672 & 0.2979 & -0.1831 & 1.0423 & -0.0696 & 0.0749 & 0.3922 & 0.9761 \\
& $(0.0175)$ & $(0.0308)$ & $(0.0336)$ & $(0.0484)$ & $(0.0788)$ & $(0.0120)$ & $(0.0094)$ & $(0.0096)$ & $(0.0114)$ \\
\hline
\end{tabular}

Note: The parameter shift for either $\alpha$ or $\gamma$ occurs in the middle of the sample. Each entry represents the mean value over 1000 replications. The numbers in the parenthesis are the standard deviations of the estimates.

As we observed, under both cases, neglecting the parameter shifts in the data generating process (in the conditional variance equation) of realized $\mathrm{GARCH}$ can substantially overestimate the persistence. This observation is consistent with the findings established for the conventional GARCH models in Hillebrand (2005). For example, in the $\alpha$-switch case, the true overall persistence should be 0.751 (with 0.526 in regime 1 and 0.976 in regime 2). However, the estimated persistence is 0.9501 . This positive bias on persistence could potentially produce misleading information on the return and volatility distributions and dynamics. For instance, in the $\alpha$-switch case, for the first half of the data (low-persistence regime), neglecting the parameter shifts leads to an underestimation of return variance by $23.47 \%$ and an overestimation of return kurtosis by $33.16 \% .5$

Motivated from the above observation, we design the second group of Monte Carlo simulations. The true DGP is the same as in the first group, which follows (14). We investigate the performance of the proposed persistence-driven TRGARCH model under the parameter-shift DGP. In other words, we examine whether the proposed threshold structure can identify and capture the regime switch caused by a single change-point in parameters. As mentioned earlier, the threshold variable used in this paper is not directly observed. In each simulation round, an additional step is needed for construction of the persistence before proceeding to the model estimation stage. In particular, after simulating the sequence of $\left\{y_{t}\right\}$ under (14), the realized persistence of volatility is sequentially constructed via (13) following Hansen and Lunde (2014) based on rolling windows.

\footnotetext{
${ }^{4}$ In this simulation exercise, we also experiment with other values on $\alpha$ and $\gamma$. The general results are similar. To save space, we do not report those results in this paper.

${ }^{5}$ This implies that the "spurious almost integration" could have impacts on the distribution of the returns, and consequently deteriorate the corresponding risk analysis. In the empirical analysis section, we conduct a Value-at-Risk back-testing analysis for a further illustration.
} 
In this group of simulations, we set the rolling window size to be 252 , which is roughly equivalent to the number of yearly trading days in the real life. A multiple IV estimator is used for each rolling window. The number of IV's is set to be 5.6 A two-regime TRGARCH model is then fitted to the simulated data with the following regime-indicator function, $s\left(\widehat{\rho}_{t-1} \leq \rho *\right)=1$ and $s\left(\widehat{\rho}_{t-1}>\rho *\right)=2$. In this group of simulations, we set the threshold at a fixed level (0.53 in the $\alpha$ switch case or 0.65 in the $\gamma$ switch case). It is worth mentioning that in the third group of Monte Carlo experiments, we design a sequential data-driven algorithm to search for the "optimal" threshold level.

The estimation results for the second group of experiments are presented in Table 2. In general, the proposed persistence-driven TRGARCH model can successfully capture the regime switch due to the parameter shift. In both cases, the parameter estimates are close to the true values in the DGP of (14). In particular, the threshold model detects that the $\alpha$ parameter is shifted from 0.1173 to 0.5392 and the $\gamma$ parameter is shifted from 0.1206 to 0.3865 , while the true parameter jumps are from 0.1 to 0.5 (for $\alpha$ ) and from 0.1 to 0.4 (for $\gamma$ ) respectively. In addition, based on the parameter estimates, the implied persistence (constructed from Proposition 1) is also close to the true persistence in the DGP. For instance, in the $\alpha$ switch case, the true persistence for each regime should be 0.526 and 0.976 respectively. The corresponding estimated ones are 0.5704 and 0.9637 . In summary, with an appropriate threshold level, the proposed algorithm can identify the regime-switch caused by parameter shifts.

In the third group of Monte Carlo experiments, we investigate a data-driven algorithm to search for the "optimal" threshold level of persistence. In particular, rather than fixing the threshold at an arbitrary level (for example, the mean or median), we consider each realized value of persistence as a potential candidate threshold level. In other words, an estimation is conducted for each candidate threshold value. The "optimal" threshold is determined from all estimation results based on certain criterion. In this paper, we choose the "optimal" threshold level that gives the largest maximum likelihood value. In the first sub-group of simulations, we still follow the $\alpha$-switch DGP in (14) to generate the experimental data. Then, the flexible threshold estimation procedure is applied to search for the "optimal" threshold level. Since the computational cost is relatively high in this group of simulations, we set the replication number to be 2007 The results are presented in Table 3. In general, the mean estimates over these 200 replications are fairly close to the benchmark values in the DGP. The proposed data-driven algorithm finds the "optimal" threshold level (0.6457) and at the "optimal" threshold level, the parameter-shift effect is revealed in the estimates. In the true DGP, $\alpha$ is shifted from 0.10 to 0.55 with all other parameters the same under both regimes. The estimation results imply that $\alpha$ is switched from 0.1627 to 0.5454 with all other parameters' estimates close to the the true values. The last two rows in Table 3 provide the true persistence under each regime and the corresponding estimated persistence, which are quite similar as well.

\footnotetext{
${ }^{6}$ Different sizes of rolling window and different numbers of IV's are also experimented in this group of Monte Carlo simulations. No significantly different results are observed. Hence, those results are not reported in the paper to save space. They are available upon request.

${ }^{7}$ Even with 200 times replications, we need to perform 200,000 optimizations in total if we treat each value as a candidate threshold level.
} 


\section{Empirical Data Analysis}

In this section, the proposed model and estimation procedure are implemented using empirical data. In particular, two groups of empirical data are investigated in this paper. The first one is the identical data set that was used in Hansen et al. (2012). 8 In Hansen et al. (2012), for all 29 sample series, a commonly high volatility persistence is detected using the RGARCH structure. We re-visit this identical data set for a further investigation using our proposed framework. The results are presented in Table 4. For comparison, each sample is estimated under RGARCH, TRGARCH and realized heterogeneous autoregressive GARCH (RHARGARCH) 9 specifications 10 For RGARCH (1,1), there are 8 parameters, $\left(\lambda_{1}, \alpha_{1}, \gamma_{1}, \beta_{1,1}, \beta_{1,2}, \tau_{1,1}, \tau_{1,2}, \sigma_{1}\right) . \pi$ and $\phi$ represent volatility persistence and leverage effect. For the TRGARCH $(1,1)$, there are 16 unknowns, which are $\left(\lambda_{1}, \lambda_{2}, \alpha_{1}, \alpha_{2}, \gamma_{1}, \gamma_{2}, \beta_{1,1}, \beta_{2,1}, \beta_{1,2}, \beta_{2,2}, \tau_{1,1}, \tau_{2,1}, \tau_{1,2}, \tau_{2,2}, \sigma_{1}, \sigma_{2}\right) . \pi$ is the overall volatility persistence $\left(\pi_{1}\right.$ and $\pi_{2}$ measure the volatility persistence for regime 1 and 2 respectively). $\phi$ presents the overall leverage effect $\left(\phi_{1}\right.$ and $\phi_{2}$ represent the leverage effect in regime 1 and 2 respectively). Lastly, $\rho *$ is the "optimal" volatility persistence threshold level found by the proposed algorithm. We also report the average volatility level under each regime in the two columns of (vol1 and vol2) in Table 4. For RHARGARCH $(1,1)$, there are 10 unknowns, which are $\left(\lambda_{1}, \alpha_{1}, \gamma_{1}, \beta_{1,1}, \delta_{1}, \beta_{1,2}, \delta_{2}, \tau_{1,1}, \tau_{1,2}, \sigma_{1}\right)$. Compared to the RGARCH setting, the extra two parameters capture the information from the last week and last month. This can be generally viewed as the long-run volatility factor component in the latent volatility process, see Engle et al. (2013). In particular, following Engle et al. (2013) and Huang et al. (2016), we adopt the HAR specification as follows,

$$
h_{t}=\lambda+\alpha_{1} h_{t-1}+\gamma_{1} y_{t-1}+\frac{\delta_{1}}{5} \sum_{j=1}^{5} y_{t-j}+\frac{\delta_{2}}{22} \sum_{j=1}^{22} y_{t-j}
$$

where $\delta_{1}$ and $\delta_{2}$ capture the weekly and monthly effects in the volatility dynamic process. The empirical estimates for these two parameters are reported in the $\delta_{1}$ and $\delta_{2}$ column respectively. It is worth noting that in the RHARGARCH specification, the overall volatility persistence can be described in $\alpha_{1}+\beta_{1,2}\left(\gamma_{1}+\delta_{1}+\delta_{2}\right)$. This is reported in the $\pi$ column of Table 4 .

The first notable finding is that, in general, a relatively low volatility persistence regime is detected based on the estimation results of the TRGARCH in Table 4. However, the overall volatility persistence and leverage effect implied by TRGARCH are quite similar to those from RGARCH and RHARGARCH. It is interesting to observe that the estimates from both RGARCH and RHARGARCH are quite close to the set of estimates in the high-persistence regime for the TRGARCH. In other words, under the proposed persistence-threshold structure, an "overlooked" relatively low volatility persistence regime is identified. Moreover, based on vol1 and vol2, we find that in the high persistence regime, the average volatility level is always higher than that in the low persistence regime. The average of the mean volatility level in the regime 1 (low persistence regime) across all 29 assets is 1.25 , while in the regime 2 (high persistence regime) it is 2.94 . In

\footnotetext{
${ }^{8}$ In Hansen et al. (2012), there are 29 assets in total over the sample period from January 1, 2002 to December 31, 2007, which consists AA, AIG, AXP, BA, BAC, C, CAT, CVX, DD, DIS, GE, GM, HD, IBM, INTC, JNJ, JPM, KO, MCD, MMM, MRK, MSFT, PG, T, UTX, VZ, WMT, XOM and SPY. Please refer to Hansen et al. (2012) for the detailed summary statistics.

${ }^{9}$ Adopting the RHARGARCH model for comparisons as the RHARGARCH is designed for capturing the long memory feature in the volatility process, see Corsi (2009) and Huang et al. (2016).

${ }^{10}$ In Table 4 X-1 stands for the estimation results under RGARCH $(1,1)$ for company X. X-2 stands for the estimation results under TRGARCH $(1,1)$ for company X. X-3 stands for the estimation results under RHARGARCH $(1,1)$ for company $\mathrm{X}$.
} 
other words, the volatility level in the high persistence regime is generally $135 \%$ higher than that in the low persistence regime under our framework. This also provides consistent empirical evidence to support the findings in Ning et al. (2015). The results of RHARGARCH are consistent with those from Huang et al. (2016). In particular, we also find the RHARGARCH has much lower $\alpha_{1}$ values than RGARCH and TRGARCH as the volatility movement can be partially explained in the weekly and monthly factors by $\delta_{1}$ and $\delta_{2}$. In general, the daily effect estimate is larger than the weekly effect estimate, and followed by the monthly one. We also report the partial likelihood and full likelihood values of the three specifications across all 29 assets. It is interesting to note that the partial likelihood values of the returns are similar across these three models. However, in general, we find that the TRGARCH provides the largest likelihood values, followed by RHARGARCH and then RGARCH. This general finding supports the threshold structure in the volatility process as we can see the improvement mainly comes from the conditional volatility component. It is also not hard to understand that the full likelihood values of RHARGARCH dominate the RGARCH because of the incorporation of the long-run volatility components in the structure 11

To give a further illustration, in the following analysis, we use one representative data series, DIS, as an example. One popularly used measure in terms of the implied persistence is the halflife of volatility shocks. This measure essentially presents the time span over which a shock to current volatility level decreases to half of its original size 12 In general, regardless of RGARCH or TRGARCH specification, the conditional variance equation can be written in the form of $h_{t}=\omega_{1}+\omega_{2} h_{t-1}+v_{t-1}$, where $\omega_{1}=\lambda+\gamma \beta_{1}, \omega_{2}=\alpha+\gamma \beta_{2}$ and $v_{t-1}=\gamma\left(\tau_{1} \epsilon_{t-1}+\tau_{2}\left(\epsilon_{t-1}^{2}-1\right)+u_{t-1}\right)$. Through a repeated substitution process, $h_{t}$ can be decomposed into two components: unconditional variance $\left(\sigma^{2}\right)$ and polynomial sequence of disturbance term $\left(\sum_{j=1}^{\infty} \omega_{2}^{j-1} v_{t-j}\right)$. Therefore, the half-life measure can be computed by solving $k$ from $\frac{1}{2}=\omega_{2}^{k-1}$. As Lamoureux and Lastrapes (1990) pointed out, this half-life measure depends solely on the persistence, which can provide some perspective on the implied persistence mis-specification. In the DIS case, under the RGARCH $(1,1)$, the estimated persistence is as high as 0.98 . The interpretation in terms of the half-life measure implies that a shock to the current volatility level of DIS would take about 35 days to diminish to half of its original size. However, under the TRGARCH $(1,1)$, two regimes are detected based on volatility persistence. In the high-persistence regime, the estimated persistence is also 0.98. While, in the low-persistence regime, the persistence is only 0.70 . Using the half-life measure, it implies a much shorter impact for the shock to volatility in this regime. It would take only 3 days for a shock to volatility to diminish to its half size.

Alternatively, following Malik et al. (2005), we plot the dynamic impulse response functions in Figure 5 for DIS to visualize the effects of decreasing in volatility persistence. The dynamic impulse response function is defined as $\Im(k)=\partial h_{t} / \partial v_{t-k}$. In particular, we plot $\Im(k)$ over 30 lags under both RGARCH and TRGARCH specifications. Consistent with the findings in the half-life measure, the high-persistence regime's (regime 2 in Figure 5) dynamic impulse function behaves similarly as RGARCH's. However, we observe a very different dynamic impulse plot in the low persistence regime in the TRGARCH model. The effect of a shock on conditional volatility drops at a much faster rate. For instance, after 10 trading days (2 weeks), a unit shock

\footnotetext{
${ }^{11}$ Including the partial likelihood and full likelihood in the comparison can us understand better where the improvement comes from.

${ }^{12}$ The half-life was originally proposed in nuclear physics to measure the survival time period of stable atoms. The measure becomes popular in econometrics as well to describe the shock effects, see Lamoureux and Lastrapes (1990), Malik et al. (2005), etc.
} 
on the current volatility has only $1.86 \%$ effect left in the regime 2 , while $25.6 \%$ is remaining in regime 1 and $29.7 \%$ is remaining in the RGARCH (without threshold-regime structure). This suggests that overlooking this regime specific characteristics could lead to a significantly different conclusion. Thus, the policy based on it could be erroneous and misleading. In addition, following Engle and Ng (1993) and Hansen et al. (2012), we plot the news impact curves for different regimes in the TRGARCH model. The news impact curve presents the response for the volatility when negative/positive news arrives. In Figure 6, we see that volatility reacts to news differently in high and low persistence regimes 13

In the second part of the empirical data analysis, we carry out further investigations of Valueat-Risk (VaR) performance with our proposed framework based on two illustrative stock indices, S\&P 500 (SPY) and Dow Jones Industrial Average (DJIA). The sample data set comes from the Realized Library provided by the Oxford-Man Institute of Quantitative Finance. The sample period covers from January 1st, 2007 to December 31st, 2015. The summary statistics of the returns and realized kernels (RK) are presented in Table 5. In particular, we report the first four basic moments, sample size and the Kolmogorov-Smirnov (K-S) test statistics. As expected, both returns exhibit heavy tails. Although the kurtosis values for the $\log (\mathrm{RK})$ are relatively closer to the benchmark, 3, the K-S tests still reject the normality at $5 \%$ level.

We fit both indices (along with the RK) to RGARCH, RHARGARCH and the proposed TRGARCH 14 The empirical estimates are provided in Table 6. The general empirical findings are similar to those in the first sample data set. A relatively low volatility persistence regime is detected under TRGARCH. Although the overall persistence is similar across these three models, overlooking the low persistence regime could provide misleading economic interpretations. For instance, without the threshold structure (RGARCH), the implied half-life of volatility is about 18 days for both S\&P 500 and DJIA. With the threshold structure (TRGARCH), in regime 2 (high persistence), the implied half-life is about 70 days for both indices, while, in regime 1 (low persistence), the implied half-life is only 12.2 and 7.6 days for S\&P 500 and DJIA respectively. As mentioned earlier, we evaluate the VaR performance for further investigation. In particular, the commonly used back-testing algorithm is carried out for the constructions of the VaR measures, which serve as one diagnostic check on the candidate models. For comparison purpose, we also include the standard GARCH model. Several standard measures are constructed for evaluation. First, we define a dummy "hit-sequence" to account for violations. A violation is said to occur if $x_{t}<V a R_{t}$. Hence,

$$
N_{t}= \begin{cases}1 & \text { if } x_{t}<\operatorname{Va} R_{t} \\ 0 & \text { otherwise }\end{cases}
$$

Given a confidence level, $c \%$ (normally $95 \%$ or $99 \%$ ), $E\left(N_{t}\right)=(1-c) T$, where $T$ is the sample size. The total number of violations (TNoV) is the sum of $N_{t}$. To address the statistical significance of the empirical violations, we follow Christoffersen (1998) to construct the likelihood-ratio (LR) test, $L R=2 \log \left[\left(\frac{\Upsilon}{1-c}\right)^{n}\left(\frac{1-\Upsilon}{c}\right)^{T-n}\right] \sim \chi_{(1)}^{2}$, where $n$ is the number of violations, $\Upsilon$ is the actual

\footnotetext{
${ }^{13}$ We have also investigated the news impact curves for all 29 samples. We did not find any stylized pattern in new impact curves for the high and low persistence regimes respectively. However, for most of the samples, the news impact curves have very different shapes in different regimes. We believe that it is worth exploring more in the future study to better understand the regime specific characteristics.

${ }^{14}$ As discussed in Section 2 (see also Hansen and Huang (2016)), in this empirical study we set $\beta_{2}$ to be one for simplified interpretations. In other words, the persistence of each regime is simply the summation of $\alpha_{s_{t}}$ and $\gamma_{s_{t}}$. It is worth mentioning that we also estimate the models without this restriction. We do not observe any significant difference since $\beta_{2}$ is empirically found closed to one.
} 
proportion of the violations (APoV) to the whole sample. The critical values are 6.635 and 3.841 respectively at the $1 \%$ and $5 \%$ significance levels. Besides number of violations, an average size of violation $(\mathrm{ASoV})$ measure is used for evaluation, where $A S V=\frac{x_{t}-V a R_{t}}{V a R_{t}}$ given $N_{t}=1$. We also construct the quadratic loss function (see Lopez (1998)) to take into account the magnitude of the violations. We refer this measure as the sum squared of violations (SSoV), SSoV $=\sum_{t} S_{t}$, where $S_{t}=1+\left(x_{t}-V a R_{t}\right)^{2}$ if $x_{t}<\operatorname{Va} R_{t}$.

In Figure 7, we plot the return time series and its corresponding VaR measures at 5\% level (upper panel-SPY and lower panel-DJIA). As expected, the standard GARCH provides a relatively flatter VaR "curve". The RGARCH, RHARGARCH and TRGARCH perform similarly in the VaR measures. We expect a better performance in TRGARCH since it could accommodate/capture regime specific volatility dynamics, and thus it can present a better conditional distribution for returns than the RGARCH and RHARGARCH. In Table 7 , we present TNoV along with the corresponding LR statistics, APoV, ASoV and SSoV measures. Since the length of both indices is 2259, at 5\% level, the expected number of violations (ENoV) should be 100.35. We observe that TRGARCH provides the closest TNoV to the expected number of violations among the three candidates. From the LR test statistics, for both SPY and DJIA, we can not reject the null hypothesis that "TNoV equals ENoV" at either $5 \%$ or 1\%. GARCH performs the worst. It indicates 136 and 134 violations for SPY and DJIA respectively. Under both cases, the null hypothesis is rejected at $1 \%$ level. TRGARCH performs relatively similar to RGARCH and RHARGARCH. However, for DJIA case, we reject the null hypothesis at $5 \%$ level with both RGARCH and RHARGARCH violations. The APoV measure should be close to 5\%. As expected, TRGARCH dominates the other two. The ASoV and SSoV measures take the magnitudes of the violations into account. We do observe that the threshold structure in the realized GARCH improves these two VaR measurements under both SPY and DJIA cases. Overall, we conclude that GARCH performs the worst in terms of all VaR loss measures used in this paper. The extension by introducing the threshold effect improves the VaR performance. The underlying reason for this improvement can be also implied from the last measure in Table 7 . We construct the partial likelihood (PL) for returns (only) under each model (see Hansen et al. (2012)). We find that the TRGARCH dominates the other two with the maximum partial log-likelihood values. This indicates that the structure under threshold improves the fitting of return distribution in general.

Similar to RGARCH, the proposed TRGARCH is also a complete model. This important feature provides us a convenient way to conduct feasible multi-step-ahead forecasting on both realized measure and latent conditional volatility. The procedure of multi-step-ahead prediction based on the TRGARCH is a straightforward extension from Hansen et al. (2012). In general, from (13), we are able to construct volatility persistence sequence, based on which we could determine the regime or state of the next period volatility process. As the state $(s)$ is determined, the prediction basically follows the procedure in Hansen et al. (2012). In particular, for $p=q=1$, a $k$-period-ahead prediction can be constructed as follows,

$\left[\begin{array}{l}\hat{h}_{t+k} \\ \hat{y}_{t+k}\end{array}\right]=\left[\begin{array}{cc}\alpha_{s, 1} & \gamma_{s, 1} \\ \beta_{s, 2} \alpha_{s, 1} & \beta_{s, 2} \gamma_{s, 1}\end{array}\right]^{k}\left[\begin{array}{c}\hat{h}_{t} \\ \hat{y}_{t}\end{array}\right]+\sum_{j=0}^{k-1}\left[\begin{array}{cc}\alpha_{s, 1} & \gamma_{s, 1} \\ \beta_{s, 2} \alpha_{s, 1} & \beta_{s, 2} \gamma_{s, 1}\end{array}\right]^{j}\left(\left[\begin{array}{c}\lambda_{s} \\ \beta_{s, 1}+\beta_{s, 2} \lambda_{s}\end{array}\right]+\left[\begin{array}{c}0 \\ \tau\left(\epsilon_{t+k-j}\right)+u_{t+k-j}\end{array}\right]\right)$

Based on the in-sample estimates, we perform the out-of-sample volatility forecasts (with GARCH, RGARCH, RHARGARCH and TRGARCH) for the next 100 days. The results are plotted in Figure 8 (upper panel - SPY and lower panel - DJIA). The realized kernel is used as the 
benchmark for comparison. Overall, RGARCH, RHARGARCH and TRGARCH performs similarly (by taking realized volatility measures in the structure), while all perform uniformly better than GARCH, which produces pretty flat forecasts of volatility. To evaluate the empirical forecasting performance, we consider three commonly used measures: the $R^{2}$-measure from the Mincer-Zarnowitz (MZ) regression $\left(\log \left(r k_{t}\right)=b_{1}+b_{2} h_{t}+e_{t}\right)$, the mean squared error (MSE) measure $\left(M S E=\frac{1}{n} \sum_{t=1}^{n}\left(r k_{t}-\exp \left(h_{t}\right)\right)^{2}\right)$ and the mean absolute error (MAE) measure $\left(M A E=\frac{1}{n} \sum_{t=1}^{n}\left|r k_{t}-\exp \left(h_{t}\right)\right|\right)$. We find the $R^{2} \mathrm{~s}$ from the MZ regression for GARCH, RGARCH, RHARGARCH and TRGARCH are $65.0 \%, 70.5 \%, 70.7 \%$ and $71.2 \%$ for SPY and $60.1 \%, 65.5 \%, 65.6 \%$ and $66.3 \%$ for DJIA. The MSEs for GARCH, RGARCH, RHARGARCH and TRGARCH are 0.4021, 0.3701, 0.3692 and 0.3528 for SPY and 0.4250, 0.3919, 0.3873, 0.3712 for DJIA 15 Lastly, the MAE measures for GARCH, RGARCH, RHARGARCH and TRGARCH are $0.4367,0.4198,0.4087,0.3922$ for SPY and 0.4216, 0.4118, 0.4099, 0.4091 for DJIA. In general, one can see that the TRGARCH provides the best volatility forecasts in terms of all the measures. This is consistent with our expectation that the threshold model introduces regime specific characteristics and thus provides a flexible structure to well explain the dynamics of the volatility. Moreover, we find that the bigger the differences of the volatility persistence between the regimes, the better the performance of the proposed TRGARCH model. This is consistent with our expectation that if the two regimes are distinct in terms of volatility persistence, it would be advantageous to adopt our proposed threshold structure 16

\section{Conclusion}

In this paper, to investigate the "spurious almost integration" effect of volatility, we propose to introduce the threshold effect in the dynamics of the realized GARCH volatility process. General closed form solutions of moment conditions are available, which provides a convenient way to theoretically investigate the statistical properties of the "spurious almost integration" effect and its impacts. Furthermore, we propose to use the realized volatility persistence as the threshold candidate to trigger the regime-switch. We find that the proposed framework avoids the overestimation of the persistence due to structure breaks/paramter shifts. In the meantime, the regime specific characteristics can be well captured. Thus, the proposed model provides a better economic representation of the volatility dynamics than the conventional models. Several groups of Monte Carlo simulations have been conducted to demonstrate the performance of the proposed model and the designed estimation algorithm. In particular, the data-driven threshold search algorithm captures the true regime-switch threshold level well. In the empirical study, two sample data sets are used for illustration of the proposed framework. In general, we show that overlooking the relatively low persistence regime could lead to some misleading conclusions (such as volatility half-life measures and its dynamic impulse response). Evaluating the VaR and forecasting performance, we find that incorporating the threshold structure improves the fit on the return distribution and thus provides more accurate VaR measures and better volatility forecasts than the conventional alternatives especially when the volatility persistence is detected significantly

\footnotetext{
${ }^{15}$ We also perform the Diebold-Mariano (DM) test on the forecasting MSE measures. We found that for SPY the DM test rejects all four models; and for DJIA, the DM test rejects GARCH, RGARCH and RHARGARCH (cannot reject TRGARCH) at $5 \%$ level.

${ }^{16}$ We also perform the DM test over all 29 assets across different forecasting horizons. In general, we find that our proposed threshold model provides the best performance against the alternatives. In particular, our proposed model has more obvious advantage when the differences of the volatility persistence between the regimes are large, for example, DIS. Since the forecasting is not the focus of the paper, to save space, we do not report all these detailed results here. We leave those for further extended research based on the current work.
} 
different across regimes.

Lastly, we want to point out several important issues remaining of interest in this paper. The data-driven algorithm is designed to search for the "optimal" threshold level. However, the computational cost is high if we treat every point as one candidate threshold level. It would be beneficial to develop a more efficient way to estimate the "optimal" threshold level. The proposed model in this paper can be, in theory, applied using any threshold variables. In this paper, we address the use of the volatility persistence. It would be interesting to investigate other alternatives (or combination of multiple candidates). In the paper, for simplicity and for our economic interpretations, we set the number of regimes to be two (high versus low persistence regimes). It would be also interesting to extend the empirical study under multiple regimes. We will leave these for future research.

Acknowledgement: We wish to thank the comments from the participants in the Western Economics 50th Anniversary Conference, the Econometrics Workshop at University of Guelph, the Econometric Society Asian Meeting and Econometric Society China Meeting. We would like to acknowledge the International Research Partnership Grant at the University of Waterloo for the financial support of this project.

\section{Notes}

\section{Appendix}

\section{A Proofs}

\section{Proof of Proposition 1.}

Suppose the probability/propotion in regime $k$ is defined as $p_{k}=p\left(s_{\rho}=s_{k}\right)$. Without loss of generality, we focus on regime $k$. Substituting the $k$ th equation in (44) into the corresponding $k$ th equation in (5), when $p=1$ and $q=1$, we have,

$$
h_{t}=\lambda_{s_{k}}+\alpha_{s_{k}} h_{t-1}+\gamma_{s_{k}} \beta_{s_{k}, 1}+\gamma_{s_{k}} \beta_{s_{k}, 2} h_{t-1}+\gamma_{s_{k}} \tau_{s_{k}, 1} \epsilon_{t-1}+\gamma_{s_{k}} \tau_{s_{k}, 2}\left(\epsilon_{t-1}^{2}-1\right)+\gamma_{s_{k}} u_{s_{k}, t-1}
$$

It could be simplified as,

$$
h_{t}=\omega_{s_{k}, 1}+\omega_{s_{k}, 2} h_{t-1}+v_{s_{k}, t-1}
$$

where $\omega_{s_{k}, 1}=\lambda_{s_{k}}+\gamma_{s_{k}} \beta_{s_{k}, 1}, \omega_{s_{k}, 2}=\alpha_{s_{k}}+\gamma_{s_{k}} \beta_{s_{k}, 2}$ and $v_{s_{k}, t-1}=\gamma_{s_{k}}\left(\tau_{s_{k}, 1} \epsilon_{t-1}+\tau_{s_{k}, 2}\left(\epsilon_{t-1}^{2}-1\right)+u_{s_{k}, t-1}\right)$. By construction, $\omega_{s_{k}, 2}$ captures the $k$ th regime persistence. Therefore the overall persistence $\omega=$ $p_{1} \omega_{s_{1}, 2}+\ldots+p_{k} \omega_{s_{k}, 2}$. This is the expression in (무).

In the $k$ th regime,

$$
\begin{aligned}
\varphi_{k}=\operatorname{corr}\left(\epsilon_{t}, v_{s_{k}, t}\right) & =\frac{E\left[\epsilon_{t} \gamma_{s_{k}}\left(\tau_{s_{k}, 1} \epsilon_{t}+\tau_{s_{k}, 2}\left(\epsilon_{t}^{2}-1\right)+u_{s_{k}, t}\right)\right]}{\sqrt{\operatorname{var}\left(v_{s_{k}, t}\right)}} \\
& =\frac{\tau_{s_{k}, 1}}{\sqrt{\tau_{s_{k}, 1}^{2}+2 \tau_{s_{k}, 2}^{2}+\sigma_{s_{k}}^{2}}}
\end{aligned}
$$


Therefore, the overall leverage effect, $\varphi_{k}=p_{1} \varphi_{1}+\ldots+p_{k} \varphi_{k}$, which is the expression in (7).

\section{Proof of Proposition 2.}

Without loss of generality, we focus on the $k$ th regime.

(A) From the proof in Proposition 1, we have $h_{t}=\omega_{s_{k}, 1}+\omega_{s_{k}, 2} h_{t-1}+v_{s_{k}, t-1}$. By a repeated substitution of $h$-expression on the right-hand side, this equation can be transformed into,

$$
h_{t}=\frac{\omega_{s_{k}, 1}}{1-\omega_{s_{k}, 2}}+\sum_{j=0}^{\infty} \omega_{s_{k}, 2}^{j} v_{s_{k}, t-j-1}
$$

Therefore, the MGF of $h$ can be derived as follows, in the $k$ th regime,

$$
\begin{aligned}
M_{h_{k}}(\eta) & =E(\exp (\eta h))=E\left(\exp \left(\eta \frac{\omega_{s_{k}, 1}}{1-\omega_{s_{k}, 2}}\right) \exp \left(\eta \sum_{j=0}^{\infty} \omega_{s_{k}, 2}^{j} v_{s_{k}, t-j-1}\right)\right) \\
& =\exp \left(\eta \frac{\omega_{s_{k}, 1}}{1-\omega_{s_{k}, 2}}\right) \prod_{j=0}^{\infty} M_{v}\left(\eta \omega_{s_{k}, 2}^{j}\right)
\end{aligned}
$$

where the MGF for $v$ is defined as follows.

$$
\begin{aligned}
M_{v}(\xi) & =E(\exp (\xi v))=E\left(\exp \left(\xi \gamma_{s_{k}}\left(\tau_{s_{k}, 1} \epsilon_{t-1}+\tau_{s_{k}, 2}\left(\epsilon_{t-1}^{2}-1\right)+u_{s_{k}, t-1}\right)\right)\right) \\
& =M_{\epsilon}\left(\xi \gamma_{s_{k}} \tau_{s_{k}, 1}\right) M_{u}\left(\xi \gamma_{s_{k}}\right) \exp \left(-\xi \gamma_{s_{k}} \tau_{s_{k}, 2}\right) M_{\epsilon^{2}}\left(\xi \gamma_{s_{k}} \tau_{s_{k}, 2}\right) \\
& =\exp \left(-\xi \gamma_{s_{k}} \tau_{s_{k}, 2}+\frac{1}{2} \xi^{2} \gamma_{s_{k}}^{2}\left(\tau_{s_{k}, 1}^{2}+\sigma_{s_{k}}^{2}\right)\right)\left(1-2 \xi \gamma_{s_{k}} \tau_{s_{k}, 2}\right)^{-\frac{1}{2}}
\end{aligned}
$$

Therefore, the MGF for $h$ can be expressed as,

$$
M_{h}(\eta)=\sum_{k=1}^{K} p_{k} \exp \left(\frac{\eta \omega_{s_{k}, 1}}{1-\omega_{s_{k}, 2}}\right) \prod_{j=0}^{\infty} M_{v}\left(\eta \omega_{s_{k}, 2}^{j}\right) .
$$

(B) Since in the $k$ th regime, $y_{t}=\beta_{s_{k}, 1}+\beta_{s_{k}, 2} h_{t}+\tau_{s_{k}, 1} \epsilon_{t}+\tau_{s_{k}, 2}\left(\epsilon_{t}^{2}-1\right)+u_{s_{k}, t}$, we have,

$$
\begin{aligned}
M_{y}(\eta) & =E\left(e^{\eta y}\right)=E\left(e^{\eta\left(\beta_{s_{k}, 1}+\beta_{s_{k}, 2} h_{t}+\tau_{s_{k}, 1} \epsilon_{t}+\tau_{s_{k}, 2}\left(\epsilon_{t}^{2}-1\right)+u_{s_{k}, t}\right)}\right) \\
& =\exp \left(\eta\left(\beta_{s_{k}, 1}-\tau_{s_{k}, 2}\right)\right) M_{h}\left(\eta \beta_{s_{k}, 2}\right) M_{\epsilon}\left(\eta \tau_{s_{k}, 1}\right) M_{\epsilon^{2}}\left(\eta \tau_{s_{k}, 2}\right) M_{u}(\eta)
\end{aligned}
$$

Since $\epsilon$ and $u$ are normally distributed, the above can be simplified to,

$$
\exp \left(\eta\left(\beta_{s_{k}, 1}-\tau_{s_{k}, 2}\right)+\frac{1}{2} \eta^{2}\left(\tau_{s_{k}, 1}^{2}+\sigma_{s_{k}}^{2}\right)\right)\left(1-2 \eta \tau_{s_{k}, 2}\right)^{-\frac{1}{2}} M_{h_{k}}\left(\eta \beta_{s_{k}, 2}\right)
$$

This expression can be viewed as the product of three MGFs, $M_{\zeta_{k}}(\eta) M_{\epsilon^{2}}(\eta) M_{h_{k}}\left(\eta \beta_{s_{k}, 2}\right)$, where $\zeta_{k} \sim$ $N\left(\beta_{s_{k}, 1}-\tau_{s_{k}, 2}, \tau_{s_{k}, 1}^{2}+\sigma_{s_{k}}^{2}\right)$.

Hence, the MGF for $y$ is $\sum_{k=1}^{K} p_{k} M_{\zeta_{k}}(\eta) M_{\epsilon^{2}}(\eta) M_{h_{k}}\left(\eta \beta_{s_{k}, 2}\right)$.

\section{Proof of Proposition 3.}

$$
\begin{aligned}
E\left(x_{t}^{m}\right) & =E\left(e^{\frac{m}{2} h_{t}} \epsilon_{t}^{m}\right) \\
& =\frac{\partial^{m} M_{\epsilon}(\eta)}{\partial \eta^{m}} \|_{\eta=0}\left(\sum_{k=1}^{K} p_{k} M_{h_{k}}\left(\frac{m}{2}\right)\right)
\end{aligned}
$$


where $M_{h_{k}}($.$) is defined in the proof of Proposition 2$ and $\frac{\partial^{m} f(\xi)}{\partial \xi^{m}} \|_{\xi=0}$ stands for the $m$ th derivative of $f($.$) w.r.t \xi$ evaluated at $\xi=0$.

More generally,

$$
E\left(x_{t}^{m} x_{t+g}^{n}\right)=E\left(e^{\frac{m}{2} h_{t}} e^{\frac{n}{2} h_{t+g}} \epsilon_{t}^{m} \epsilon_{t+g}^{n}\right)
$$

Since, in regime $k, h_{t}=\omega_{s_{k}, 1}+\omega_{s_{k}, 2} h_{t-1}+v_{s_{k}, t-1}$, via iterative substitutions, we have $h_{t+g}=\omega_{s_{k}, 2}^{g} h_{t}+$ $\omega_{s_{k}, 1} \sum_{j=1}^{g} \omega_{s_{k}, 2}^{j-1}+\sum_{j=1}^{g} \omega_{s_{k}, 2}^{g-j} v_{t+g-j}$. Hence,

$$
\begin{aligned}
E\left(x_{t}^{m} x_{t+g}^{n}\right) & =E\left[\exp \left(\left(\frac{m+n \omega_{s_{k}, 2}^{g}}{2}\right) h_{t}\right) \exp \left(\frac{n}{2} \omega_{s_{k}, 1} \sum_{j=1}^{g} \omega_{s_{k}, 2}^{j-1}\right) \exp \left(\frac{n}{2} \sum_{j=1}^{g} \omega_{s_{k}, 2}^{g-j} v_{t+g-j}\right)\right] \\
& \times \frac{\partial^{m} M_{\epsilon}(\eta)}{\partial \eta^{m}}\left\|_{\eta=0} \frac{\partial^{n} M_{\epsilon}(\eta)}{\partial \eta^{n}}\right\|_{\eta=0}
\end{aligned}
$$

In the $k$ th regime (refer to the proof in Proposition 2),

$$
\begin{aligned}
M_{h_{k}}(\xi) & =\exp \left(\frac{\xi \omega_{s_{k}, 1}}{1-\omega_{s_{k}, 2}}\right) \prod_{j=0}^{\infty} M_{v}\left(\xi \omega_{s_{k}, 2}^{j}\right) \\
M_{v}(\xi) & =\exp \left(-\xi \gamma_{s_{k}} \tau_{s_{k}, 2}+\frac{1}{2} \xi^{2} \gamma_{s_{k}}^{2}\left(\tau_{s_{k}, 1}^{2}+\sigma_{s_{k}}^{2}\right)\right)\left(1-2 \xi \gamma_{s_{k}} \tau_{s_{k}, 2}\right)^{-\frac{1}{2}}
\end{aligned}
$$

Therefore,

$$
\begin{aligned}
& E\left[\exp \left(\left(\frac{m+n \omega_{s_{k}, 2}^{g}}{2}\right) h_{t}\right) \exp \left(\frac{n}{2} \omega_{s_{k}, 1} \sum_{j=1}^{g} \omega_{s_{k}, 2}^{j-1}\right) \exp \left(\frac{n}{2} \sum_{j=1}^{g} \omega_{s_{k}, 2}^{g-j} v_{t+g-j}\right)\right] \\
& =\left[\sum_{k=1}^{K} p\left(s(\rho)=s_{k}\right) M_{h_{k}}\left(\frac{m+n \omega_{s_{k}, 2}^{g}}{2}\right) \exp \left(\frac{n}{2} \omega_{s_{k}, 1} \sum_{j=1}^{g} \omega_{s_{k}, 2}^{j-1}\right) \prod_{j=1}^{g} M_{v}\left(\frac{n}{2} \omega_{s_{k}, 2}^{(g-j)}\right)\right]
\end{aligned}
$$

Combining the $m$ th and $n$th derivatives of the MGF of $\epsilon$, this yields the closed-form expression of the $(m, n)$ th order cross-moment of $x_{t}$ and $x_{t+g}$.

\section{B Tables and Figures}


Figure 1: Theoretical Return Variance Evolution Under the Changes of Model Parameters
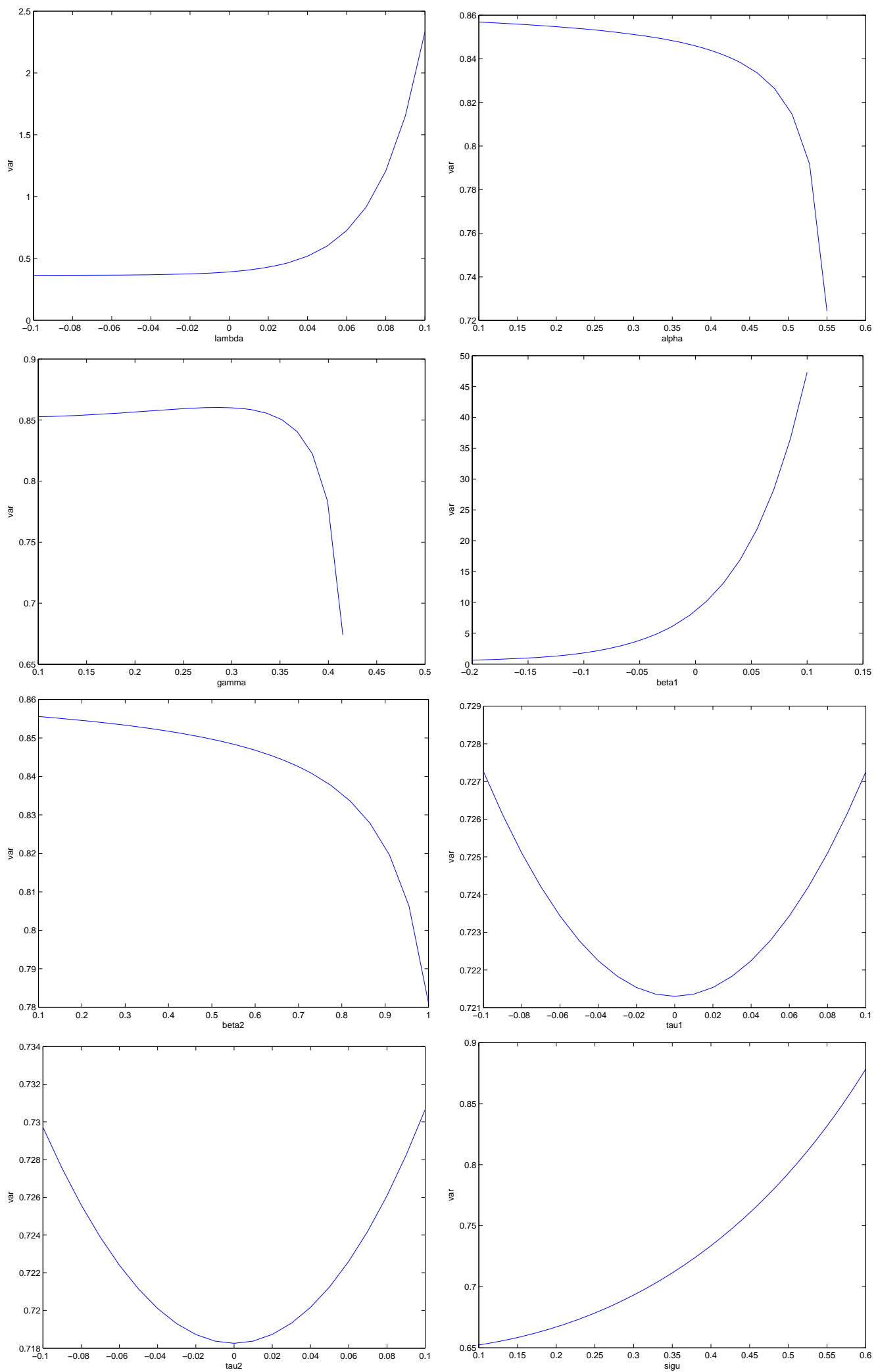
Figure 2: Theoretical Return Kurtosis Evolution Under the Changes of Model Parameters
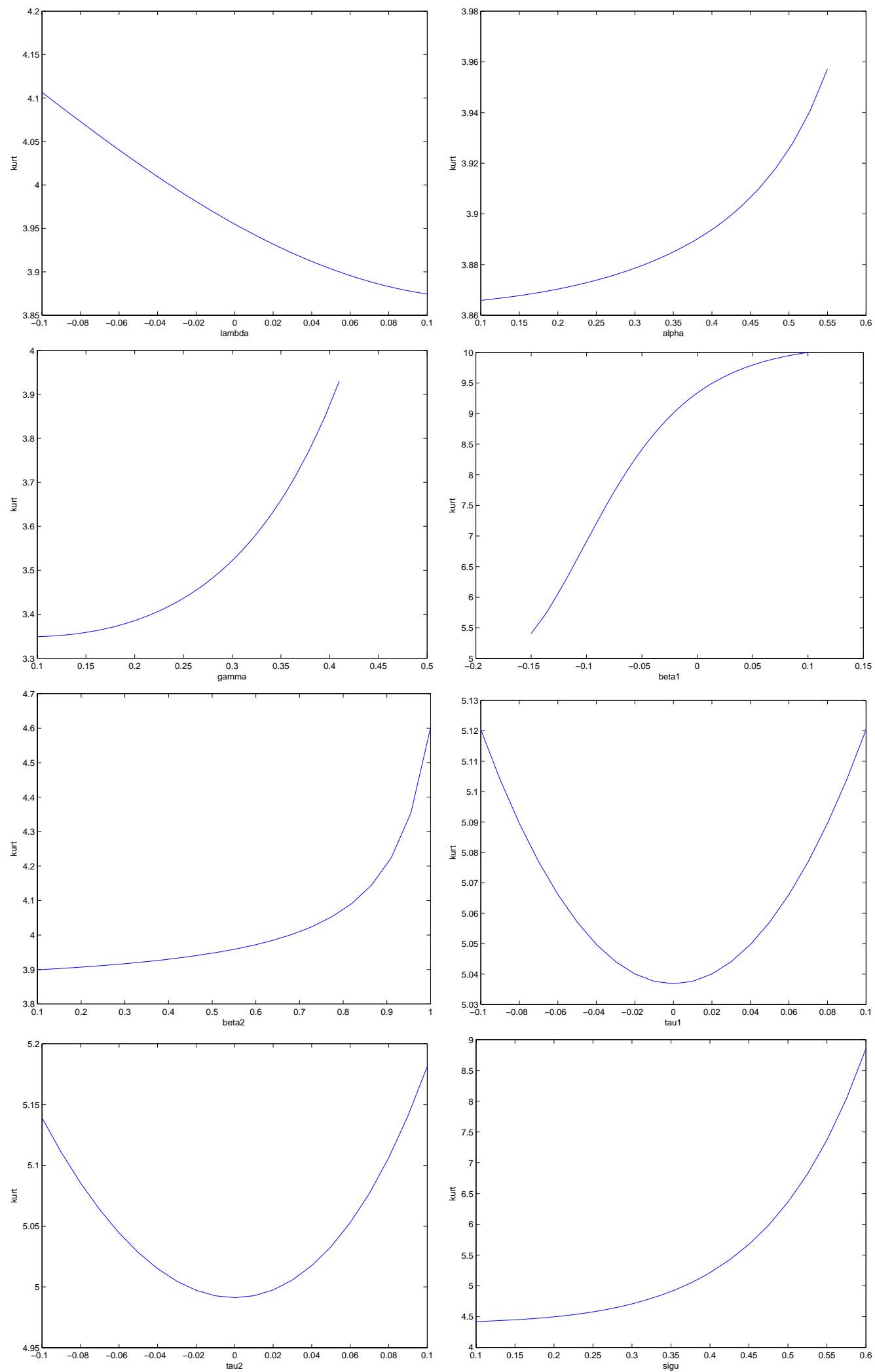
Figure 3: Persistence Parameters on Return Variance and Kurtosis
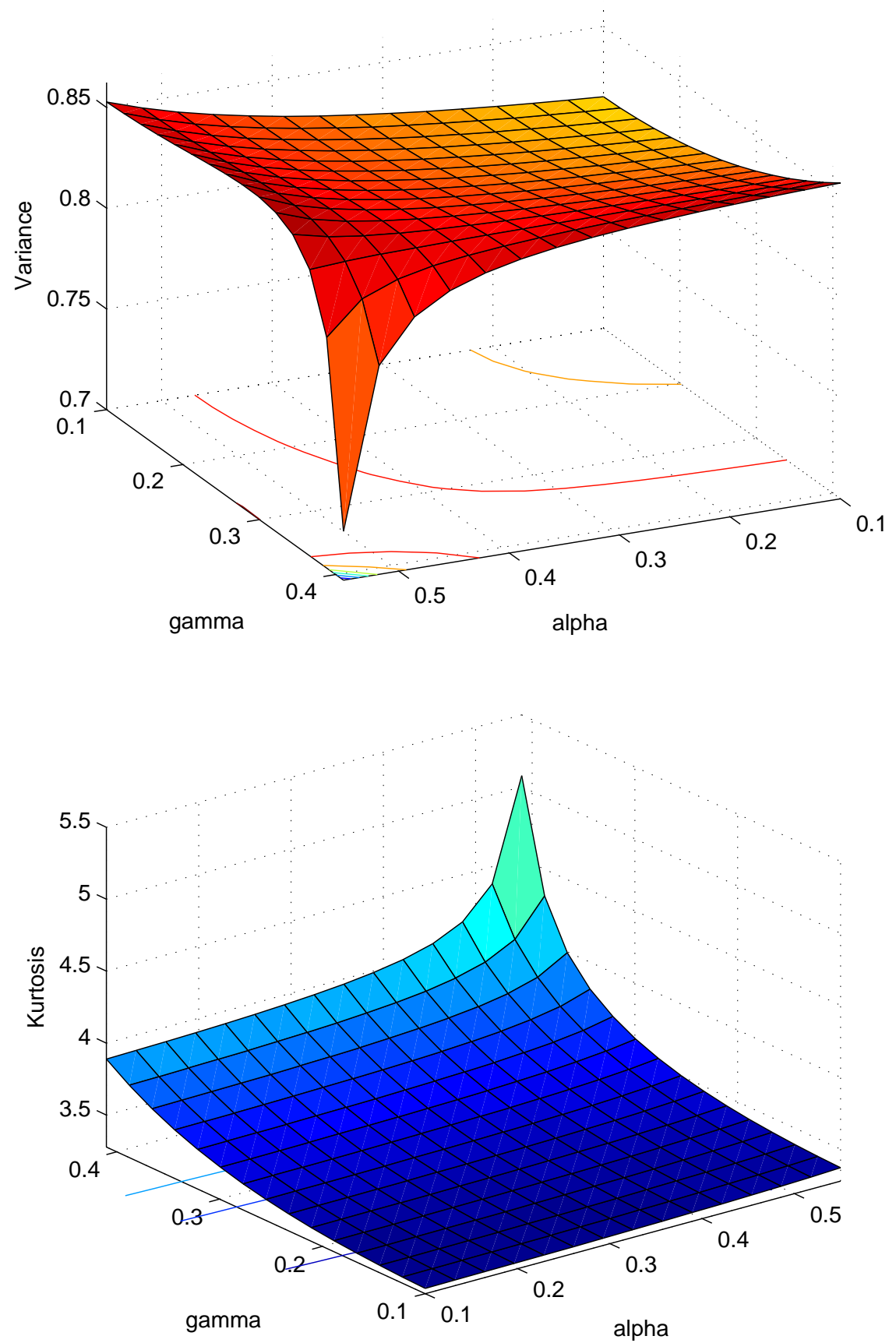
Figure 4: ACF Plots Under $\alpha$-switch and $\gamma$-switch

$\alpha$-Switch

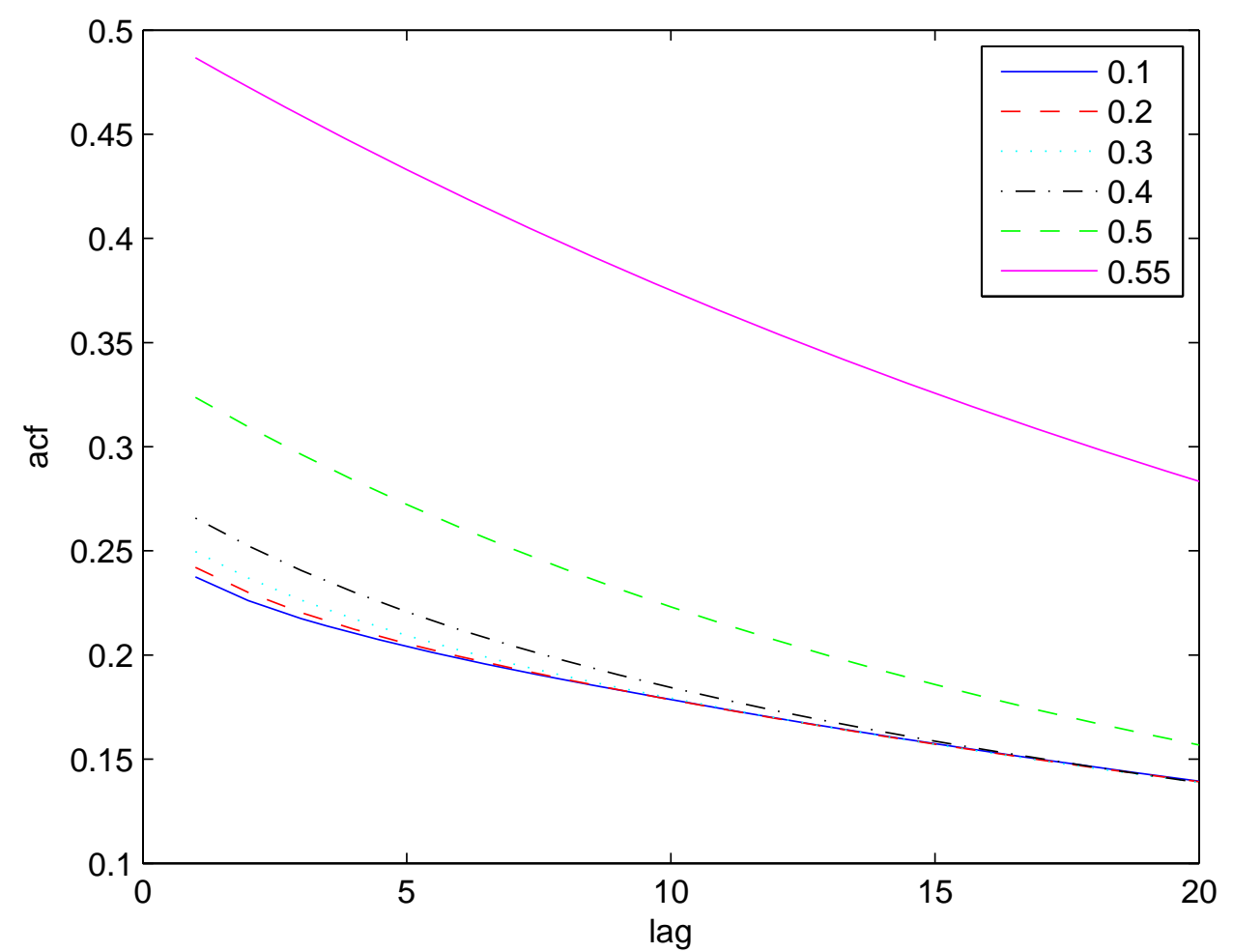

$\gamma$-Switch

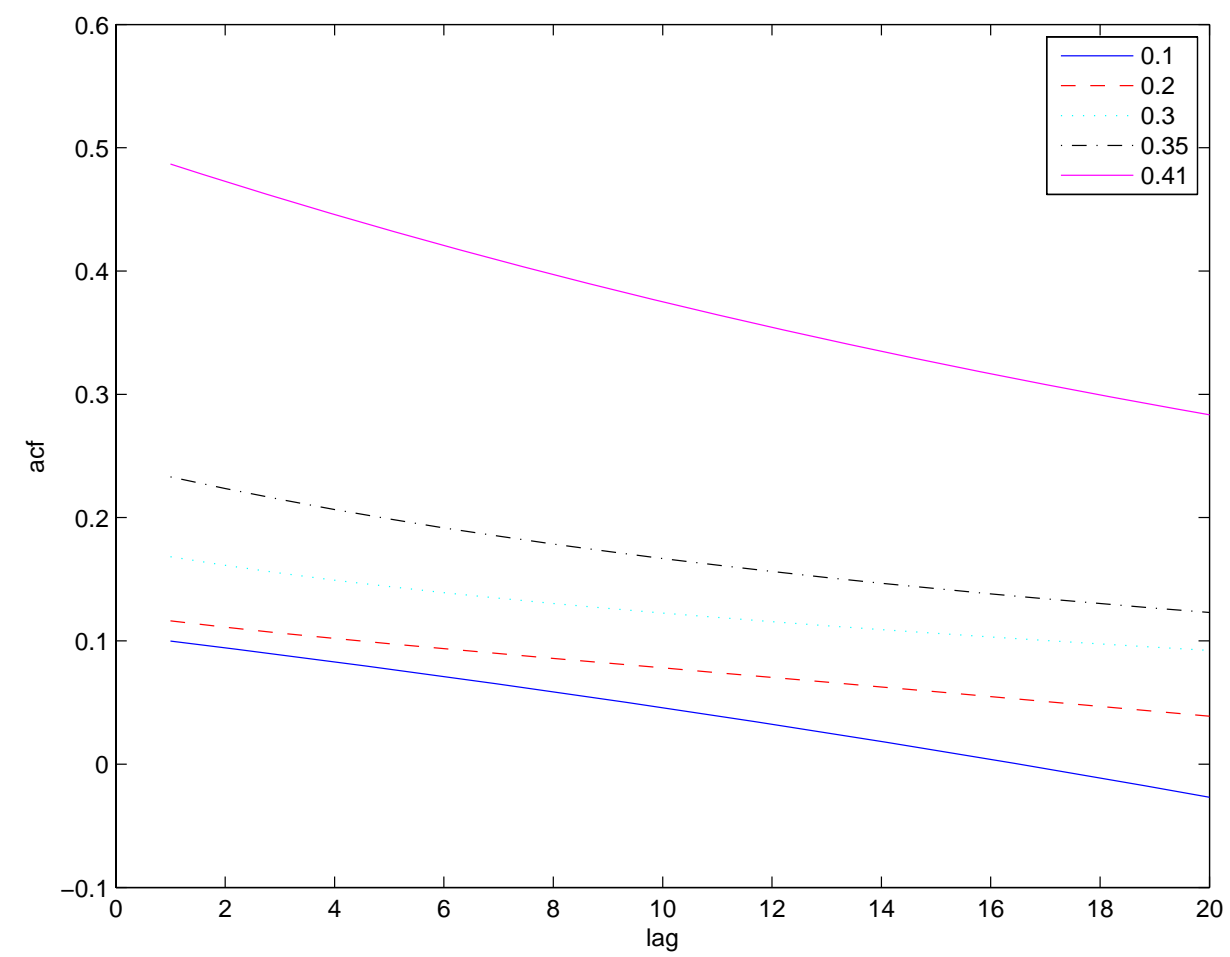

Upper panel is the $\alpha$-switch impact on ACF $(\alpha=\{0.1,0.2,0.3,0.4,0.5,0.55\})$. Lower panel is the $\gamma$-switch impact on ACF $(\gamma=\{0.1,0.2,0.3,0.35,0.41\})$. 
Table 2: Persistence-Driven realized threshold GARCH under a single parameter shift

\begin{tabular}{|c|c|c|}
\hline & $\alpha=0.10$ to 0.55 & $\gamma=0.10$ to 0.41 \\
\hline$\lambda_{1}$ & $\begin{array}{c}0.0577 \\
(0.0679)\end{array}$ & $\begin{array}{c}0.0603 \\
(0.0379)\end{array}$ \\
\hline$\lambda_{2}$ & $\begin{array}{c}0.0581 \\
(0.0341)\end{array}$ & $\begin{array}{c}0.0532 \\
(0.0358)\end{array}$ \\
\hline$\alpha_{1}$ & $\begin{array}{c}0.1173 \\
(0.0531)\end{array}$ & $\begin{array}{c}0.5591 \\
(0.1723)\end{array}$ \\
\hline$\alpha_{2}$ & $\begin{array}{c}0.5392 \\
(0.0403)\end{array}$ & $\begin{array}{c}0.5685 \\
(0.0473)\end{array}$ \\
\hline$\gamma_{1}$ & $\begin{array}{c}0.4123 \\
(0.1078)\end{array}$ & $\begin{array}{c}0.1206 \\
(0.0659)\end{array}$ \\
\hline$\gamma_{2}$ & $\begin{array}{c}0.4082 \\
(0.0471)\end{array}$ & $\begin{array}{c}0.3865 \\
(0.0520)\end{array}$ \\
\hline$\beta_{1,1}$ & $\begin{array}{l}-0.1758 \\
(0.0885)\end{array}$ & $\begin{array}{l}-0.1661 \\
(0.0638) \\
\end{array}$ \\
\hline$\beta_{2,1}$ & $\begin{array}{l}-0.1794 \\
(0.0681)\end{array}$ & $\begin{array}{l}-0.1752 \\
(0.0760)\end{array}$ \\
\hline$\beta_{1,2}$ & $\begin{array}{c}1.1077 \\
(0.2441)\end{array}$ & $\begin{array}{c}0.9442 \\
(0.2480)\end{array}$ \\
\hline$\beta_{2,2}$ & $\begin{array}{c}1.0399 \\
(0.0875)\end{array}$ & $\begin{array}{c}1.0424 \\
(0.0891)\end{array}$ \\
\hline$\tau_{1,1}$ & $\begin{array}{l}-0.0698 \\
(0.0303) \\
\end{array}$ & $\begin{array}{l}-0.0692 \\
(0.0176) \\
\end{array}$ \\
\hline$\tau_{2,1}$ & $\begin{array}{l}-0.0701 \\
(0.0156)\end{array}$ & $\begin{array}{l}-0.0693 \\
(0.0172)\end{array}$ \\
\hline$\tau_{1,2}$ & $\begin{array}{c}0.0690 \\
(0.0220) \\
\end{array}$ & $\begin{array}{c}0.0728 \\
(0.0136) \\
\end{array}$ \\
\hline$\tau_{2,2}$ & $\begin{array}{c}0.0728 \\
(0.0114)\end{array}$ & $\begin{array}{c}0.0710 \\
(0.0123)\end{array}$ \\
\hline$\sigma_{1}$ & $\begin{array}{c}0.3757 \\
(0.0207)\end{array}$ & $\begin{array}{c}0.3834 \\
(0.0138)\end{array}$ \\
\hline$\sigma_{2}$ & $\begin{array}{c}0.3859 \\
(0.0117)\end{array}$ & $\begin{array}{c}0.3815 \\
(0.0129)\end{array}$ \\
\hline$\pi_{1}$ & $\begin{array}{c}0.5704 \\
(0.1570)\end{array}$ & $\begin{array}{c}0.6730 \\
(0.2046)\end{array}$ \\
\hline$\pi_{2}$ & $\begin{array}{c}0.9637 \\
(0.0185)\end{array}$ & $\begin{array}{c}0.9714 \\
(0.0135) \\
\end{array}$ \\
\hline
\end{tabular}

Note: The parameter shift for either $\alpha$ or $\gamma$ occurs in the middle of the sample. Each entry represents the mean value over 1000 replications. The numbers in the parenthesis are the standard deviations of the estimates. 
Table 3: Persistence-Driven realized threshold GARCH under a single parameter shift

\begin{tabular}{r|c|c}
\hline & true parameter value & estimates \\
\hline$\lambda_{1}$ & 0.06 & $0.0573(0.0303)$ \\
\hline$\lambda_{2}$ & 0.06 & $0.0536(0.0281)$ \\
\hline$\alpha_{1}$ & 0.10 & $0.1627(0.0812)$ \\
\hline$\alpha_{2}$ & 0.55 & $0.5454(0.0400)$ \\
\hline$\gamma_{1}$ & 0.41 & $0.4108(0.1202)$ \\
\hline$\gamma_{2}$ & 0.41 & $0.4086(0.0507)$ \\
\hline$\beta_{1,1}$ & -0.18 & $-0.1681(0.0823)$ \\
\hline$\beta_{2,1}$ & -0.18 & $-0.1715(0.0832)$ \\
\hline$\beta_{1,2}$ & 1.04 & $1.1563(0.4454)$ \\
\hline$\beta_{2,2}$ & 1.04 & $1.0402(0.0928)$ \\
\hline$\tau_{1,1}$ & -0.07 & $-0.0709(0.0262)$ \\
\hline$\tau_{2,1}$ & -0.07 & $-0.0713(0.0166)$ \\
\hline$\tau_{1,2}$ & 0.07 & $0.0702(0.0154)$ \\
\hline$\tau_{2,2}$ & 0.07 & $0.0708(0.0139)$ \\
\hline$\sigma_{1}$ & 0.38 & $0.3803(0.0372)$ \\
\hline$\sigma_{2}$ & 0.38 & $0.3782(0.0109)$ \\
\hline$\pi_{1}$ & 0.5704 & $0.5980(0.1010)$ \\
\hline$\pi_{2}$ & 0.9637 & $0.9670(0.0171)$ \\
\hline
\end{tabular}

Note: The parameter shift for $\alpha$ occurs in the middle of the sample. Each entry represents the mean value over 200 replications. The numbers in the parenthesis are the standard deviations of the estimates. The mean of the optimal threshold level is 0.6457 . 


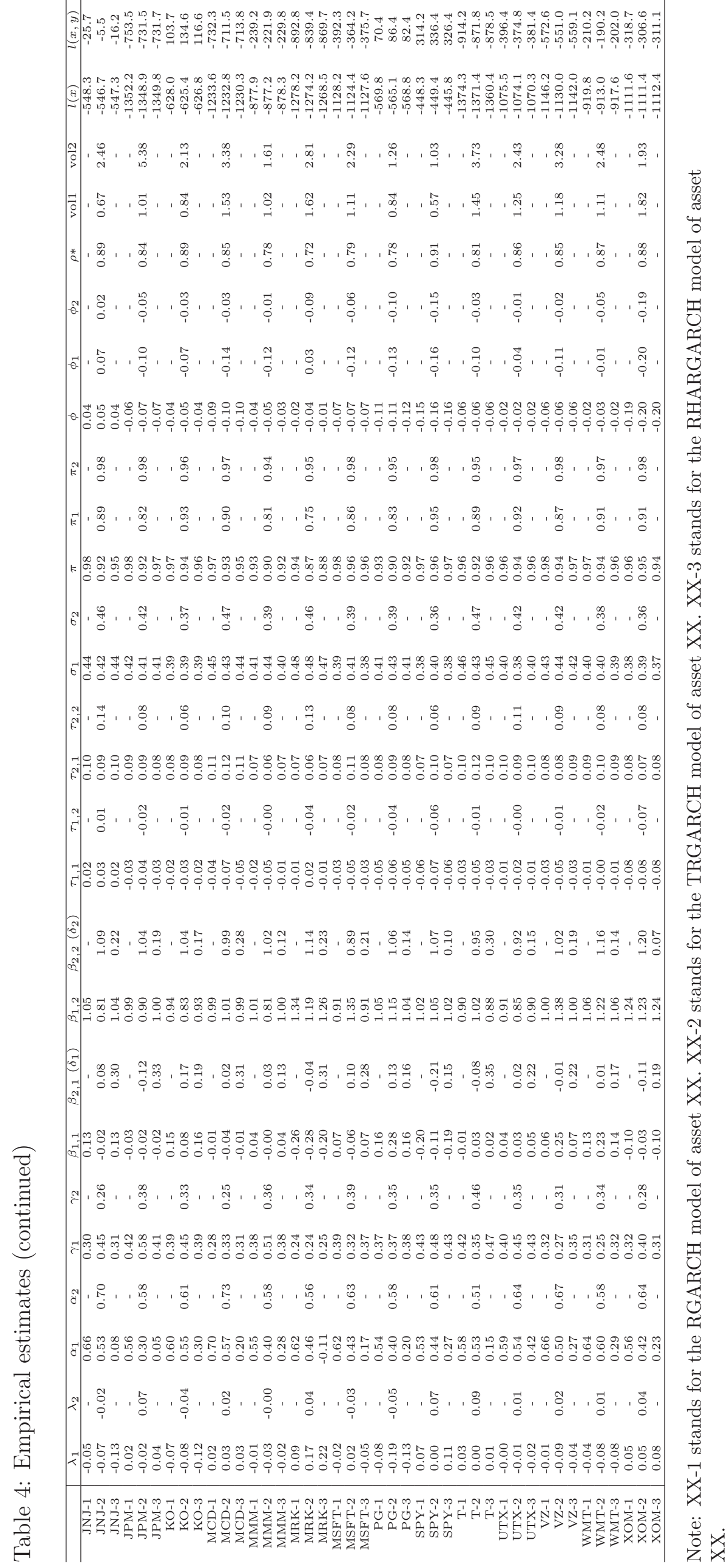


Figure 5: Dynamic Impulse Function (DIS)

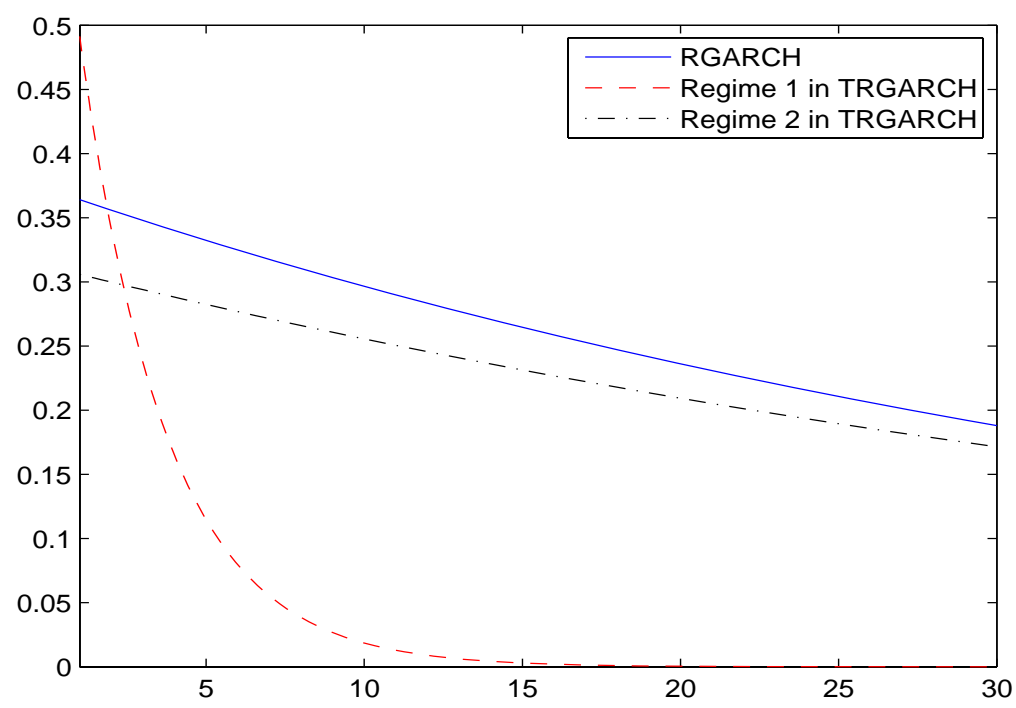

Figure 6: News Impact Curve (DIS)

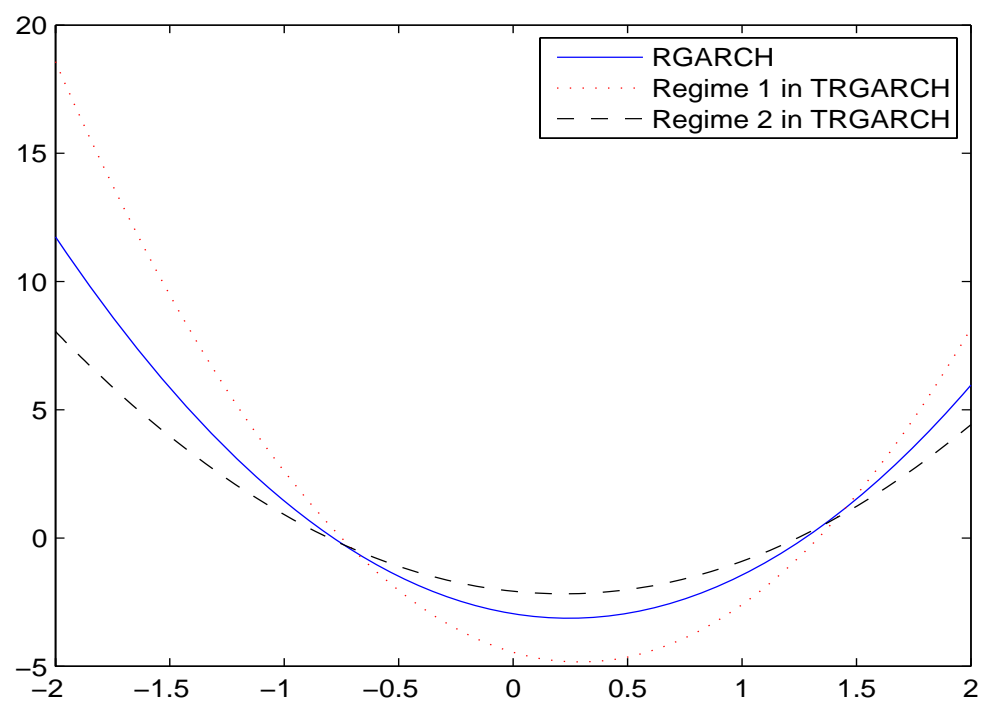


Table 5: Summary Statistics

\begin{tabular}{l||c|c|c|c|c|c}
\hline & Mean & Var & Skew & Kurt & Length & K-S test \\
\hline SPY-Return & 0.0182 & 1.6313 & -0.2701 & 12.3270 & 2259 & 0.1068 \\
\hline SPY-Log(RK) & -0.5601 & 1.2903 & 0.6251 & 3.5710 & 2259 & 0.0408 \\
\hline DJIA-Return & 0.0243 & 1.5056 & 0.0518 & 12.7276 & 2259 & 0.1052 \\
\hline DJIA-Log(RK) & -0.5480 & 1.2331 & 0.6855 & 3.6952 & 2259 & 0.0452 \\
\hline
\end{tabular}

Note: K-S test stands for the Kolmogorov-Smirnov Normality test at $5 \%$ level. The corresponding critical value is 0.0285 .

Table 6: Empirical Estimates - SPY and DJIA

\begin{tabular}{|c|c|c|c|c|c|c|}
\hline & SPY-1 & SPY-2 & SPY-3 & DJIA-1 & DJIA-2 & DJIA-3 \\
\hline$\lambda_{1}$ & $0.19(0.0854)$ & $0.21(0.0670)$ & $0.28(0.0014)$ & $0.14(0.0171)$ & $0.16(0.0900)$ & $0.21(0.0014)$ \\
\hline$\lambda_{2}$ & - & $0.14(0.0578)$ & - & $0.11(0.0046)$ & & \\
\hline$\alpha_{1}$ & $0.48(0.0633)$ & $0.42(0.0156)$ & $0.27(0.0054)$ & $0.54(0.0352)$ & $0.42(0.1203)$ & $0.37(0.0087)$ \\
\hline$\alpha_{2}$ & - & $0.62(0.1121)$ & - & $0.63(0.0330)$ & & \\
\hline$\gamma_{1}$ & $0.48(0.0719)$ & $0.52(0.0069)$ & $0.48(0.0007)$ & $0.42(0.0288)$ & $0.48(0.0739)$ & $0.41(0.0008)$ \\
\hline$\gamma_{2}$ & - & $0.36(0.1001)$ & - & $0.35(0.0310)$ & & \\
\hline$\beta_{1,1}$ & $-0.41(0.0893)$ & $-0.43(0.0762)$ & $-0.41(0.070)$ & $-0.35(0.0540)$ & $-0.41(0.1827)$ & $-0.35(0.008)$ \\
\hline$\beta_{2,1}$ & - & $-0.38(0.0206)$ & - & $-0.31(0.0371)$ & & \\
\hline$\tau_{1,1}$ & $-0.12(0.0087)$ & $-0.13(0.0031)$ & $-0.12(0.0001)$ & $-0.10(0.0017)$ & $-0.11(0.0086)$ & $-0.11(0.0001)$ \\
\hline$\tau_{2,1}$ & - & $-0.11(0.0294)$ & - & $-0.11(0.0045)$ & & \\
\hline$\tau_{1,2}$ & $0.13(0.0135)$ & $0.12(0.0055)$ & $0.12(0.0001)$ & $0.15(0.0093)$ & $0.14(0.0113)$ & $0.14(0.001)$ \\
\hline$\tau_{2,2}$ & - & $0.16(0.0080)$ & - & $0.17(0.0047)$ & & \\
\hline$\sigma_{1}$ & $0.51(0.0034)$ & $0.52(0.0002)$ & $0.51(0.0011)$ & $0.53(0.0007)$ & $0.52(0.0012)$ & $0.52(0.0081)$ \\
\hline$\sigma_{2}$ & - & $0.48(0.0010)$ & - & $0.52(0.0003)$ & & \\
\hline$\delta_{1}$ & - & - & $0.09(0.0054)$ & - & - & $0.08(0.0081)$ \\
\hline$\delta_{2}$ & - & - & $0.11(0.0005)$ & - & - & $0.10(0.0006)$ \\
\hline$\pi_{1}$ & 0.96 & 0.94 & 0.96 & 0.96 & 0.90 & 0.96 \\
\hline$\pi_{2}$ & - & 0.98 & - & - & 0.98 & - \\
\hline
\end{tabular}

Note: XX-1 stands for the RGARCH model of asset XX. XX-2 stands for the TRGARCH model of asset XX. XX-3 stands for the RHARGARCH model of asset XX. The numbers in the parenthesis are the standard errors of the estimates. 
Figure 7: Value-at-Risk
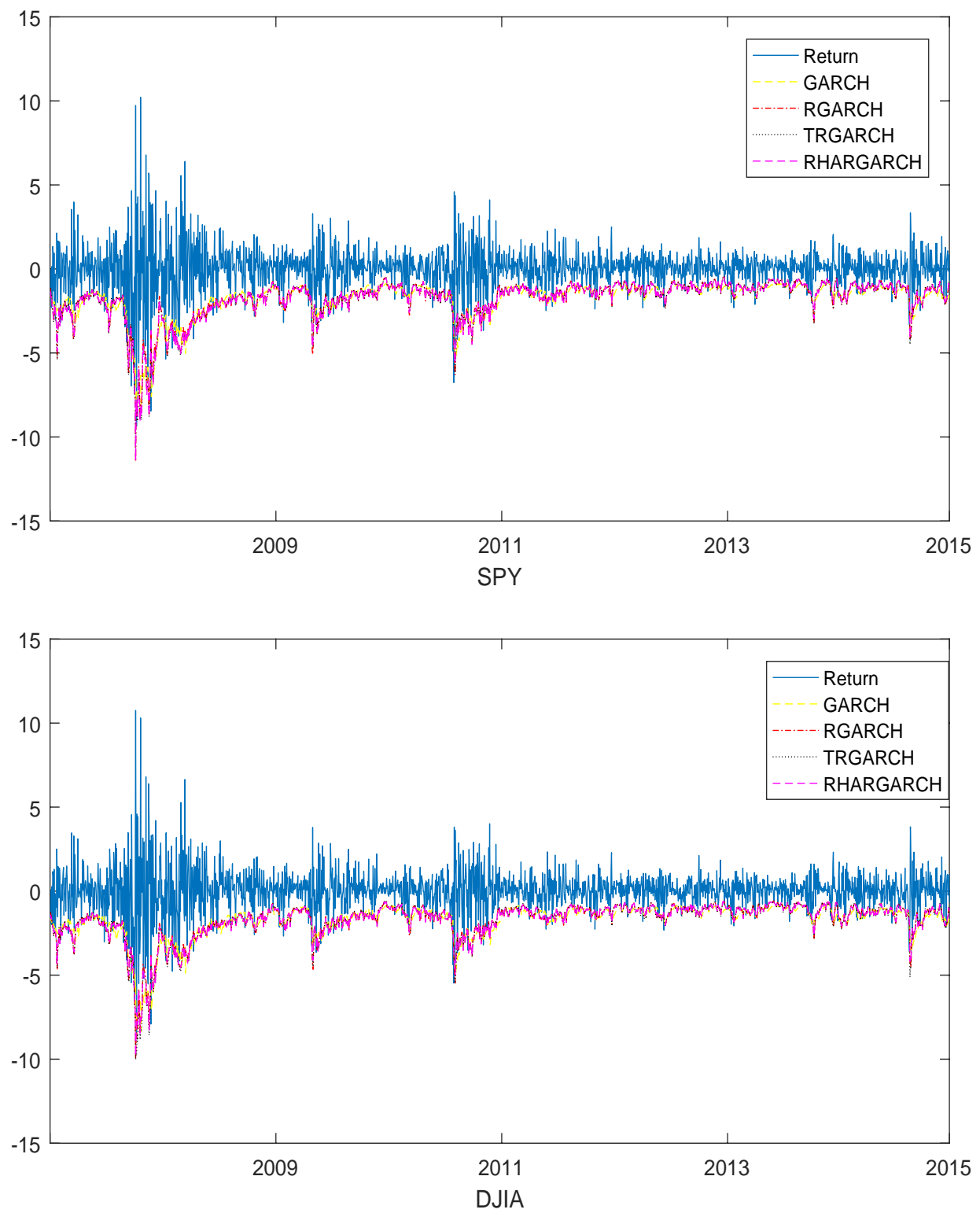
Table 7: VaR - SPY and DJIA

\begin{tabular}{c|c|c|c|c|c}
\hline & TNoV & APoV & ASoV & SSoV & PL \\
\hline SPY-(GARCH) & $136(12.06)^{* *}$ & $6.78 \%$ & $42.93 \%$ & 2.9871 & -3137.7 \\
\hline SPY-(RGARCH) & $120(3.82)$ & $5.98 \%$ & $36.29 \%$ & 1.8052 & -2701.4 \\
\hline SPY-(TRGARCH) & $118(3.10)$ & $5.88 \%$ & $35.56 \%$ & 1.7681 & -2684.3 \\
\hline SPY-(RHARGARCH) & $121(4.21)$ & $6.03 \%$ & $35.81 \%$ & 1.7781 & -2692.3 \\
\hline DJIA-(GARCH) & $134(10.80)^{* *}$ & $6.68 \%$ & $38.85 \%$ & 2.4443 & -3076.3 \\
\hline DJIA-(RGARCH) & $122(4.61)^{*}$ & $6.08 \%$ & $32.87 \%$ & 1.5734 & -2660.4 \\
\hline DJIA-(TRGARCH) & $118(3.10)$ & $5.88 \%$ & $31.28 \%$ & 1.5397 & -2651.5 \\
\hline DJIA-(RHARGARCH) & $122(4.61)^{*}$ & $6.08 \%$ & $31.50 \%$ & 1.5689 & -2655.5 \\
\hline
\end{tabular}

Expected number of violations is 100.35 for both SPY and DJIA. * and ** denotes significance at the $5 \%$ and $1 \%$ level respectively.

Figure 8: Volatility Forecast

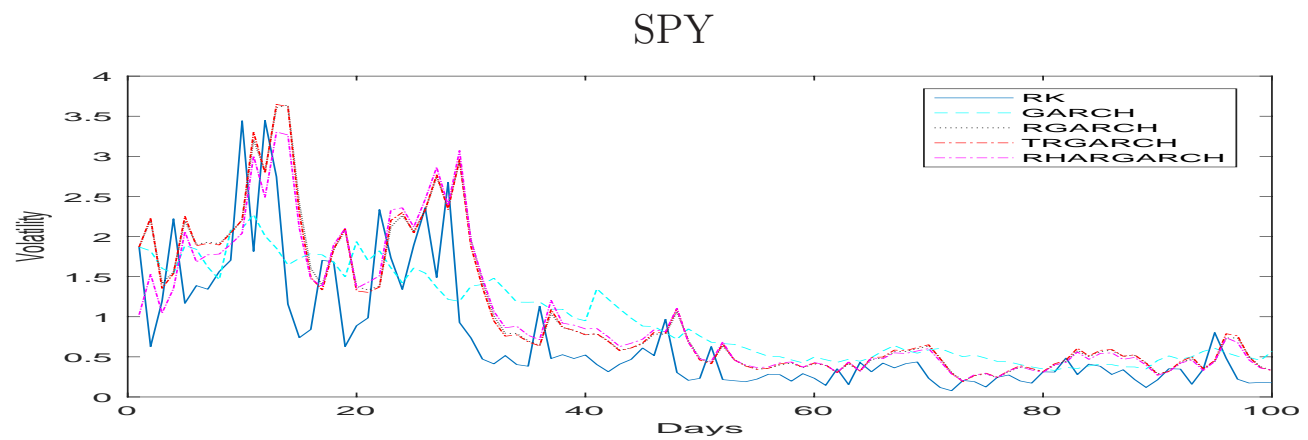

DJIA



\section{References}

Andersen, T., Bollerslev, T., Diebold, F., and Labys, P. (2003). Modelling and forecasting realized volatility. Econometrica, 71:529-626.

Barndorff-Nielsen, O., Hansen, P., Lunde, A., and Shephard, N. (2008). Designing realised kernels to measure the ex-post variation of equity prices in the presence of noise. Econometrica, 76:1481-1536. 
Barndorff-Nielsen, O., Hansen, P., Lunde, A., and Shephard, N. (2011). Multivariate realised kernels: Consistent positive semi-definite estimators of the covariation of equity prices with noise and nonsynchronous trading. Journal of Econometrics, 162:149-169.

Barndorff-Nielsen, O. and Shephard, N. (2002). Econometric analysis of realized volatility and its use in estimating stochastic volatility models. Journal of the Royal Statistical Society B, 64:253-280.

Bollerslev, T. (1986). Generalized autoregressive conditional heteroskedasticity. Journal of Econometrics, $31: 307-327$.

Bollerslev, T. and Zhou, H. (2006). A simple framework for gauging return-volatility regressions. Journal of Econometrics, 131:123-150.

Brooks, C. (2001). A doubel-threshold GARCH model for the French Franc/Deutschmark exchange rate. Journal of Forecasting, 20:135-143.

Chaussé, P. and Xu, D. (2016). GMM estimation of a realized stochstic volatility model: a Monte Carlo study. Forthcoming in Econometric Reviews.

Chen, C. and So, M. (2006). On a threshold heteroscedastic model. International Journal of Forecasting, 22:73-89.

Christoffersen, P. F. (1998). Evaluating interval forecasts. International Economic Review, 39(4):841862.

Corradi, V. and Distaso, W. (2006). Semi-parametric comparison of stochastic volatility models using realized measures. Review of Economic Studies, 73:635-667.

Corsi, F. (2009). A simple approximate long-memory model of realized volatility. The Review of Economics and Statistics, 7(2):174-196.

Diebold, F. (1986). Modeling the persistence of conditional variances: a comment. Econometric Reviews, $5: 51-56$.

Dobrev, D. and Szerszen, P. (2010). The information content of high-frequency data for estimating equity return models and forecasting risk. FEDS Working Paper No. 2010-45.

Engle, R. (1982). Autoregressive conditional heteroskedasticity with estimates of the variance of united kingdom inflation. Econometrica, 50:987-1007.

Engle, R. and Gallo, G. (2006). A multiple indicators model for volatility using intra-daily data. Journal of Econometrics, 131:3-27.

Engle, R. and Ng, V. (1993). Measuring and testing the impact of news on volatility. Journal of Finance, 48:1747-1778.

Engle, R. F., Ghysels, E., and Sohn, B. (2013). Stock market volatility and macroeconomic fundamentals. The Review of Economics and Statistics, 95:776-797.

Glosten, L. R., Jagannathan, R., and Runkle, D. E. (1993). On the relation between the expected value and the volatilty of the nominal excess retuen on stocks. Journal of Finance, 48:1779-1801.

Hamilton, J. and Susmel, R. (1994). Autoregressive conditional heteroskedasticity and changes in regimes. Journal of Econometrics, 64:307-333.

Hansen, P. and Huang, Z. (2016). Exponential GARCH modeling with realized measures of volatility. Journal of Business and Economic Statistics. 
Hansen, P., Huang, Z., and Shek, H. (2012). Realized GARCH: A joint model for returns and realized measures of volatility. Journal of Applied Econometrics, 27:877-906.

Hansen, P. and Lunde, A. (2014). Estimating the persistence and teh autocorrelation function of a time series that is meaured with error. Econometric Theory, 30:60-93.

Hillebrand, E. (2005). Neglecting parameter changes in GARCH models. Journal of Econometrics, 129:121-138.

Huang, Z., Liu, H., and Wang, T. (2016). Modeling long memory volatility using realized measures of volatility: A realized har garch model. Economic Modelling, 52:812-821.

Koopman, S. and Scharth, M. (2013). The analysis of stochastic volatility in the presence of daily realised measures. Journal of Financial Econometrics, 11:76-115.

Lamoureux, C. and Lastrapes, W. D. (1990). Persistence in variance structural change and the garch model. Journal of Business and Economic Statistics, 8:225-234.

Li, W. K. and Lam, K. (1995). Modeling asymmetry in stock returns by a threshold ARCH model. The Statistician, 44:333-341.

Lopez, J. (1998). Methods for evaluating value-at-risk estimates. FRBNY.

Malik, F., Ewing, B., and Payne, J. E. (2005). Measuing volatility persistence in the presence of sudden changes in the variance of canadian stock returns. The Canadian Journal of Economics, 38:1037-1056.

Ning, Q., Xu, D., and Wirjanto, S. T. (2015). Is volatility clustering of asset returns asymmetric? Journal of Banking and Finance, 52:62-76.

Noureldin, D., Shephard, N., and Sheppard, K. (2012). Multivariate high frequency-based volatility (HEAVY) models. Journal of Applied Econometrics, 27(6):907-933.

Shephard, N. and Sheppard, K. (2010). Realising the future: Forecasting with high-frequency-based volatility (HEAVY) models. Journal of Applied Econometrics, 25:197-231.

Straumann, D. and Mikosch, T. (2012). Quasi-maximum-likelihood estimation in conditionally heteroscedastic time series: A stochastic recurrence equations approach. Annals of Statistics, 34:24492495 .

Takahashi, M., Omori, Y., and Watanabe, T. (2009). Estimating stochastic volatility models using daily returns and realized volatility simultaneously. Computational Statistics and Data Analysis, 53:24042426.

$\mathrm{Xu}$, D. (2013). Examining realized volatility regimes under a threshold stochastic volatility model. International Journal of Finance and Economics, 17:373-389.

Zakoian, J. M. (1994). Threshold heteroskedastic models. Journal of economic Dynamic and control, 18:931-955.

Zhang, L., Mykland, P., and Ait-Sahalia, Y. (2005). A tale of two scales: Determining integrated volatility with noisy high frequency data. Journal of the American Statistical Association, 100:1394-1411. 